\title{
Cysteine peptidases of Eudiplozoon nipponicum: a broad repertoire of structurally assorted cathepsins $L$ in contrast to the scarcity of cathepsins B in an invasive species of haematophagous monogenean of common carp
}

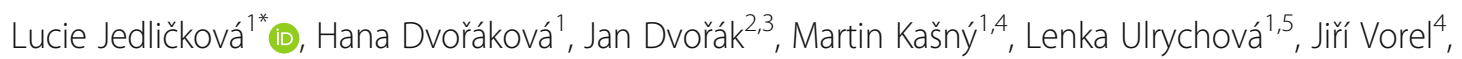
Vojtěch Žárský ${ }^{6}$ and Libor Mikeš ${ }^{1}$

\begin{abstract}
Background: Cysteine peptidases of clan CA, family C1 account for a major part of proteolytic activity in the haematophagous monogenean Eudiplozoon nipponicum. The full spectrum of cysteine cathepsins is, however, unknown and their particular biochemical properties, tissue localisation, and involvement in parasite-host relationships are yet to be explored.

Methods: Sequences of cathepsins $L$ and $B(E n C L$ and $E n C B$ ) were mined from E. nipponicum transcriptome and analysed bioinformatically. Genes encoding two EnCLs and one EnCB were cloned and recombinant proteins produced in vitro. The enzymes were purified by chromatography and their activity towards selected substrates was characterised. Antibodies and specific RNA probes were employed for localisation of the enzymes/transcripts in tissues of $E$. nipponicum adults.

Results: Transcriptomic analysis revealed a set of ten distinct transcripts that encode EnCLs. The enzymes are significantly variable in their active sites, specifically the $\$ 2$ subsites responsible for interaction with substrates. Some of them display unusual structural features that resemble cathepsins B and S. Two recombinant EnCLs had different pH activity profiles against both synthetic and macromolecular substrates, and were able to hydrolyse blood proteins and collagen I. They were localised in the haematin cells of the worm's digestive tract and in gut lumen. The EnCB showed similarity with cathepsin B2 of Schistosoma mansoni. It displays molecular features typical of cathepsins B, including an occluding loop responsible for its exopeptidase activity. Although the EnCB hydrolysed haemoglobin in vitro, it was localised in the vitelline cells of the parasite and not the digestive tract.
\end{abstract}

(Continued on next page)

\footnotetext{
*Correspondence: lucka.jedlickova@seznam.cz

1 Department of Parasitology, Faculty of Science, Charles University, Viničná 7,

12844 Prague 2, Czech Republic

Full list of author information is available at the end of the article
} 
(Continued from previous page)

Conclusions: To our knowledge, this study represents the first complex bioinformatic and biochemical characterisation of cysteine peptidases in a monogenean. Eudiplozoon nipponicum adults express a variety of CLs, which are the most abundant peptidases in the worms. The properties and localisation of the two heterologously expressed EnCLs indicate a central role in the (partially extracellular?) digestion of host blood proteins. High variability of substrate-binding sites in the set of EnCLs suggests specific adaptation to a range of biological processes that require proteolysis. Surprisingly, a single cathepsin B is expressed by the parasite and it is not involved in digestion, but probably in vitellogenesis.

Keywords: Cysteine peptidase, Protease, Cathepsin, S2 subsite, Haematophagy, Blood digestion, Monogenea, Diplozoidae, Eudiplozoon nipponicum, Fish parasite

\section{Background}

Blood-feeding monogeneans of the family Diplozoidae (Heteronchoinea) are ectoparasites that live on the gills of cyprinid fishes. One member of the family, Eudiplozoon nipponicum, is an important invasive species, first recorded in Europe in 1983 on farmed carp in France [1]. It is currently found throughout Europe and is a widespread representative of the helminth fauna of the common carp (Cyprinus carpio) in the Czech Republic [2]. The common carp is a fish of high economic importance in many Asian and European countries, with global aquaculture production yielding over 4 million tons in 2014 [3]. Since the parasite affects the health of farmed fish, although precise estimates are unknown, it is assumed it causes economic losses. Pathogenic effects of E. nipponicum are associated mainly with inducing hypochromic microcytic anaemia in the fish by a continuous intake of blood by worms attached to the carp gills [4].

Monogenea are a rather neglected group of Neodermata and only a handful of papers on their biochemistry and bioactive molecules have ever been published. Based on previous ultrastructural studies, it has been assumed that in diplozoid monogeneans, the digestion of blood, gathered by combined action of their powerful buccal suckers and muscular pharynx, takes place within the lysosomal cycle in the specialised cells of the intestinal epidermis [5-9], similarly as in blood-sucking mites such as ticks $[10,11]$. Our previous study [12] has shown that the processing of blood in E. nipponicum relies on an evolutionarily conserved multi-enzyme network of cysteine and aspartic peptidases, similar to the proteolytic cascades of other blood-feeding helminths such as Schistosoma, Fasciola, Ancylostoma, etc. [13-16]. Among the endopeptidases of E. nipponicum, clan CA cysteine peptidase activities, including cathepsin L-like and cathepsin B-like activity, are dominant [12].

In helminths, cathepsins $\mathrm{L}$ and $\mathrm{B}$ play various roles. Due to their histolytic potency, they are involved in host invasion and tissue migration, but they also play a role in various pathological processes, immune evasion, and other parasite-host interactions, as well as in helminth reproduction, nutrient digestion, etc. [17-19]. In general, peptidases encoded in the helminth genomes are mostly temporarily expressed in the various life stages, thus reflecting the parasite's specific needs regarding digestion or other hydrolytic processes. For instance, a characterisation of several cathepsins L of the liver fluke Fasciola hepatica had shown that the infective larvae use cathepsin L3 to traverse the host's intestinal wall, while the fluke's migratory stages utilise cathepsin L2 to penetrate host liver tissue, and adults employ cathepsin L1 jointly with cathepsins L2 and L5 to digest host proteins [20]. The use of different peptidases with overlapping substrate specificities helps heteroxenous and tissue-migrating parasites to adapt to various environments and sources of nutrition within the hosts. On the other hand, little is known about the complex functioning of cysteine peptidases in monoxenous blood-feeding monogeneans that spend most of their life attached to a single type of host tissue, such as the gills.

In the present study, we focused on clan CA cysteine peptidases, namely cathepsins $\mathrm{L}$ and $\mathrm{B}$, selected due to their abundance in the transcriptome of adult E. nipponicum worms. We have employed phylogenetic and bioinformatic analysis to investigate their relationship to other helminth peptidases. We have selected two of the most abundant cathepsins L and the only expressed cathepsin B, and produced these as functionally active recombinant forms using the Pichia pastoris expression system. A biochemical and functional characterisation of the recombinant enzymes was performed in order to understand their specificity and substrate preference. Furthermore, we have developed specific antibodies and RNA probes to localise proteins/transcripts within the worms' bodies by immunofluorescence assay and RNA in situ hybridisation technique. Our work thus presents the first detailed functional characterisation of monogenean peptidases.

\section{Methods}

Parasite material

Adult worms of E. nipponicum were collected from the gills of infected carps (C. carpio) freshly slaughtered in a commercial facility of the fishery Rybářství Třeboň, Plc., Třeboň basin, South Bohemia, Czech Republic. A soluble worm extract, parasite RNA, and first-strand cDNA were 
obtained as previously described [12]. For RNA in situ hybridisation, the worms were flat-fixed between microscopic slides in Bouin's solution (Sigma-Aldrich, Darmstadt, Germany) at RT for $1 \mathrm{~h}$, then transferred into fresh fixative and incubated at $4{ }^{\circ} \mathrm{C}$ overnight. For immunohistochemistry, 4\% paraformaldehyde in PBS was employed as a fixative. Fixed worms were dehydrated with increasing concentrations of ethyl alcohol (70-100\%), cleared with xylene, and embedded in paraffin.

\section{Sequence analyses of cathepsin L-like and B-like peptidases of E. nipponicum}

Transcripts from the transcriptome of adult E. nipponicum worms were assembled within a parallel project and deposited in the NCBI GenBank database under accession number GFYM00000000. They were annotated by searching for the closest homologues. We have used BLASTp and BLASTn algorithms (E-value 1e-5) to mine the following public sequence databases: NCBI non-redundant protein database [21], MEROPS database of peptidases and their inhibitors [22], UniProtKB/UniRef100 database and UniProtKB/TrEMBL only used for searching for protein sequences related to phylum Platyhelminthes (Taxon ID: 6157) [23], UniProtKB/Swiss-Prot [24], RCSB PDB [25] and DDBJ [26]. Sequences encoding cathepsin L-like and B-like peptidases of E. nipponicum were identified and their amino acid sequences (catalytic domains) aligned with sequences of formerly recognised as E. nipponicum cathepsins L, EnCL1 (GenBank: KP793605) and EnCL3 (GenBank: KP793606), F. hepatica cathepsin L1 (GenBank: AAT76664.1), $F$. hepatica cathepsin L3 (GenBank: CAC12807.1) and with E. nipponicum cathepsin B, EnCB (GenBank: MF346929), Schistosoma mansoni cathepsins B1 (GenBank: CAD44624.1) and B2 (GenBank: XP_018651608.1), respectively, using Clustal Omega tool (http://www.ebi.ac.uk/Tools/msa/clustalo/) [27]. The relative abundance of cathepsin $\mathrm{L}$ transcripts, which reflects the rate of transcription of the corresponding genes, was predicted by back mapping of raw Illumina reads to the assembled transcripts using Kallisto v. 0.43.0 [28]. The presence of a signal sequence in amino acid sequences of the enzymes was predicted by SignalP 4.1 Server (http://www.cbs.dtu.dk/services/SignalP/) [29]. A theoretical position of the pro-region and composition of the enzymes' S2 subsites of the active site were determined by multiple sequence alignment (see above). Molecular mass and theoretical pI of the deduced proteins were determined by Compute $\mathrm{pI} / \mathrm{Mw}$ software available at the ExPASy web portal (https://web.expasy.org/compute_pi/) [30]. Potential N-glycosylation and $\mathrm{O}$-glycosylation sites were identified using an online tool at NetNGlyc 1.0 and NetOGlyc 4.0 Servers (http:// www.cbs.dtu.dk/services/NetNGlyc [30] and http://www. cbs.dtu.dk/services/NetOGlyc [31]).
Eudiplozoon nipponicum cathepsin L1 and L2 amino acid sequences were used as queries for BLAST searches in the NCBI non-redundant protein database [32] and the Wormbase database of platyhelminths [33]. Recovered sequences were further analysed using CLANS software [34] and a cluster of 515 sequences containing E. nipponicum cathepsins was selected. Redundant sequences were filtered at 95\% level of identity using the CD-HIT software [35]. Divergent and incomplete sequences were manually removed, which resulted in a dataset of 288 sequences. These were aligned using the MAFFT [36] and the alignment was trimmed using BMGE (239 sites) [37]. We inferred a maximum-likelihood tree using the best-fit model $(\mathrm{LG}+\mathrm{C} 60+\mathrm{G})$, and tested the topology with 10,000 ultrafast bootstraps in IQ-TREE [38]. Bayesian inference of phylogeny was run under the C60 siteheterogeneous mixture model using Phylobayes.

\section{Expression of recombinant cathepsins L1, L3 and B in Pichia pastoris and their purification}

Two abundant EnCLs (EnCL1 and EnCL3) and the only $E n C B$ were selected for a functional expression. Genespecific primers (Additional file 1) and the first-strand cDNA of adult E. nipponicum were used to amplify the yrEnCL1 (GenBank: KP793605), yrEnCL3 (GenBank: KP793606), and $y r E n C B$ (GenBank: MF346929) genes. The amplified products were ligated into a $P$. pastoris expression vector pPICZ $\alpha$ B as described previously [39], and the constructs were verified by Sanger sequencing. Protein expression in P. pastoris X-33 was carried out following a protocol described previously [40] and according to the manual of the Easy Select Pichia Expression Kit (Thermo Fisher Scientific, Waltham, Massachusetts). The yeast medium was centrifuged and the supernatant filtered $(0.22 \mu \mathrm{m})$ and concentrated on AmiconUltra-15 filters 10,000 MWCO (Millipore, Darmstadt, Germany). The enzymes were affinity purified via their His-tag using Ni-chelating chromatography (HisTrap ${ }^{\mathrm{Tm}}$ FF crude, GE Healthcare, Little Chalfont, United Kingdom) and a stepwise elution by imidazole $(0.05-0.5 \mathrm{M})$. Yeastorigin hyperglycosylation of $E n C B$ was removed by endoglycosidase F1 (Calbiochem, Darmstadt, Germany). Purification was completed by cation exchange FPLC on Mono $\mathrm{S}^{\mathrm{m}} 5 / 50 \mathrm{GL}$ column (GE Healthcare) using $20 \mathrm{mM}$ MES buffer pH 6.0 and gradient elution by $\mathrm{NaCl}(0-1 \mathrm{M})$.

\section{Expression of recombinant cathepsin L1 in Escherichia coli} and its purification

The EnCL1 gene (GenBank: KP793605) was amplified using first-strand cDNA of adult E. nipponicum and specific primers (Additional file 2). The amplified product was ligated into the $E$. coli expression vector pet28a + (Novagen, Darmstadt, Germany) and verified by DNA sequencing. 
EnCL1 was expressed in E. coli BL21 Star ${ }^{\mathrm{ma}}$ (DE3) (Invitrogen, Carlsbad, California) according to pET System Manual (Novagen). Harvested cells were lysed in $20 \mathrm{mM}$ Tris- $\mathrm{HCl} \mathrm{pH} 8 / 0.3 \mathrm{M} \mathrm{NaCl} / 1 \%$ lauryl sarcosine/10 mM imidazole by sonication, and centrifuged at $10,000 \times \mathrm{g}$ for $10 \mathrm{~min}$. Inclusion bodies were solubilised with $6 \mathrm{M}$ guanidine hydrochloride as described elsewhere [41]. The solubilised mixture was filtered $(0.65 \mu \mathrm{m})$, passed over the column containing Ni-NTA beads (Qiagen, Hilden, Germany), and eluted by imidazole $(0.05-0.5 \mathrm{M})$. Chromatographic fractions were analysed by SDS-PAGE and the identity of suspected protein bands was verified by mass spectrometry as described in [12].

\section{Processing the $y r E n C L 3$ pro-enzyme}

Auto-processing of pro-yrEnCL3 was tested by incubation of the recombinant protein in $50 \mathrm{mM} / 100 \mathrm{mM}$ $\mathrm{CPB}$, with $2 \mathrm{mM}$ DTT at $\mathrm{pH}$ values of 4,5 and 6 . Aliquots of $20 \mu \mathrm{l}$ were taken from reactions at specific time intervals $(15,30,60$ and $120 \mathrm{~min})$ at $37^{\circ} \mathrm{C}$, whereby incubations were stopped by adding $10 \mu \mathrm{M}$ E-64. Processing of the enzyme to its mature form was monitored by SDS-PAGE, Western blotting and Edman degradation of N-terminus and subsequently confirmed by fluorometry with the corresponding synthetic peptide substrates (see below). Processing of $y r E n C L 3$ was also monitored by affinity labelling using $20 \mu \mathrm{M}$ fluorescent probe BODIPYgreen-DCG-04 [42] and $2.5 \mu \mathrm{g}$ of the enzyme (30 min incubation at $\mathrm{pH}$ 5) as described previously [40]. Specificity of the probe towards the active site of the peptidase was verified by pre-incubation with the cysteine peptidase-specific inhibitor E-64 $(10 \mu \mathrm{M})$ for 5 min.

\section{Peptidase activity assays}

Peptidase activities of recombinant cathepsins were measured with fluorogenic aminomethylcoumarin peptide substrates (Bachem) as described previously [12]. Substrates tested with $y r E n C L 1$ and $y r E n C L 3$ included Z-Phe-Arg-AMC (FR), Z-Leu-Arg-AMC (LR), Z-ArgArg-AMC (RR), Z-Pro-Arg-AMC (PR) and Z-Gly-ProArg-AMC (GPR). The $y r E n C B$ was tested with FR and RR substrates only. The purified $r E n C L s(15 \mathrm{nM})$ and $r E n C B$ (30 $\mathrm{nM}$ ) were dissolved in $100 \mu \mathrm{l}$ of $50 \mathrm{mM} / 100 \mathrm{mM} \mathrm{CPB}$ supplemented with $2 \mathrm{mM}$ DTT, in $\mathrm{pH}$ range of $3-8$. The reactions were initiated by adding the individual substrates (final concentration $50 \mu \mathrm{M}$ ) in $100 \mu \mathrm{l}$ of the same buffer at $28{ }^{\circ} \mathrm{C}$. The release of free AMC was measured at excitation and emission wavelengths of 355 and $460 \mathrm{~nm}$, respectively, in Infinite M200 fluorometer (TECAN, Männedorf, Switzerland).

For inhibition assays, three inhibitors were used (final concentration $10 \mu \mathrm{M}$, Sigma-Aldrich): E-64 (L-transepoxysuccinyl-leucylamido [4-guanidino] butane), which is a general cysteine peptidase inhibitor, iCL (Arg-LysLeu-Leu-Trp-NH2), a reversible inhibitor of cathepsin L; and CA-074 (N-[L-3-trans-propylcarbamoyloxirane-2carbonyl]-Ile-Pro-OH), which is a selective inhibitor of cathepsin B. Inhibitors were mixed with enzyme samples and incubated at $28{ }^{\circ} \mathrm{C}$ for $15 \mathrm{~min}$ prior to adding the substrates. Remaining hydrolysis rates were measured with the FR substrate (cathepsins L and B), with LR (cathepsins L), or with RR (cathepsin B) as described above.

The assay of exopeptidase activity of activated $y r E n C B$ (75 $\mathrm{nM}$ ) was carried out with benzoyl-glycinyl-histidinylleucine (Bz-Gly-His-Leu; final conc. $2 \mathrm{~mm}$ ) as a substrate, using a modified protocol [43]. The activity was measured in $50 \mathrm{mM} / 100 \mathrm{mM}$ CPB pH 4-6 containing $2 \mathrm{mM}$ DTT and $0.05 \mathrm{mg} / \mathrm{ml}$ fluorescamine.

\section{Hydrolysis of macromolecular substrates by recombinant cathepsins $\mathrm{L}$ and $\mathrm{B}$}

Processed $y r E n C L 1, y r E n C L 3$ and $y r E n C B$ (2.5 $\mu \mathrm{g}$ each) were incubated at $37{ }^{\circ} \mathrm{C}$ with $50 \mu \mathrm{g}$ of macromolecular substrates (bovine haemoglobin and albumin, human collagen I, IgG and fibrinogen; Sigma-Aldrich) dissolved in $100 \mu \mathrm{l}$ of $50 \mathrm{mM} / 100 \mathrm{mM}$ CPB with $2 \mathrm{mM}$ DTT in $\mathrm{pH}$ 4.5-6. Aliquots $(10 \mu \mathrm{l})$ for SDS-PAGE analysis in $12 \%$ gels were taken at various intervals $(0,30,60$ and $120 \mathrm{~min}$ and $16 \mathrm{~h}$ ).

\section{RNA in situ hybridisation}

$E n C L 1 / E n C L 3$ gene-specific primers (Additional file 3) and first-strand cDNA of adult E. nipponicum were used for the PCR. The amplified products were ligated into pGEM $^{\circ}$-T Easy vector (Promega, Madison, Wisconsin) and verified by DNA sequencing. The constructs were linearised and used as a template for RNA probes. Both sense and anti-sense RNA probes were synthesised in vitro (Dig RNA Labelling Kit (SP6/T7); Roche, Basel, Switzerland). In situ hybridisation was performed using a modified protocol [44]. Briefly, the sections of adult worms on slides were incubated for $19 \mathrm{~h}$ at $41{ }^{\circ} \mathrm{C}$ $(E n C L 1)$ and at $37^{\circ} \mathrm{C}(E n C L 3)$ with specific RNA probes diluted 1:100 in a hybridisation mixture $(5 \times$ SSC, $1 \times$ PBS, $50 \%$ deionised formamide, $1 \%$ Tween-20, $10 \%$ dextran sulphate $\mathrm{Mw} 500 \times, 1 \mathrm{mg} / \mathrm{ml}$ Torula yeast RNA). Detection was achieved with alkaline phosphatase-conjugated anti-digoxigenin antibodies (1:500, Roche) and Fast Red TR substrate (Sigma-Aldrich). Negative controls were incubated under the same conditions but with an antisense probe or without a probe. Specific strand RT-PCR was used for the detection of natural anti-sense transcripts occurring in the monogenean cells [45].

\section{Production of peptidase-specific polyclonal antibodies}

For the production of monospecific polyclonal anti-EnCL3 and anti-EnCB antibodies, ICR/CD1 mice (ENVIGO, 
Huntingdon, United Kingdom) were injected subcutaneously twice, at a 14-day interval, with $30 \mu \mathrm{g}$ of purified pro-yrEnCL3 or deglycosylated $y r E n C B$ in sterile saline and TitermaxGold adjuvant (Sigma-Aldrich). The mice were boosted by an intramuscular injection of purified antigens $(15 \mu \mathrm{g})$ in sterile saline after another two weeks. Anti-EnCL1 antibodies were produced in mice injected intraperitoneally with $c .50 \mu \mathrm{g}$ of $b r E n C L 1$ cut as a band from a $12 \%$ gel after SDS-PAGE. The strip of the gel containing the antigen was repeatedly and thoroughly washed in $50 \%$ methanol $/ 10 \%$ acetic acid for several hours to remove SDS, washed several times in sterile saline, and homogenised in sterile saline using a motorised pellet pestle. Two antigen deliveries were performed at an interval of 14 days. All mice were bled under deep ketamine/xylazine anaesthesia 14 days after the last injection and sera were collected by centrifugation. Control sera were taken from the same mice prior to immunisation.

\section{Western and affinity blotting}

Specificity of the anti-cathepsin antibodies produced in mice was verified on PVDF membrane immunoblots of soluble worm extracts previously separated by SDS-PAGE in $12 \%$ gels $(20 \mu \mathrm{g} / \mathrm{well})$. The experiment was performed according to a modified protocol [40]. The membranes were first incubated for $1 \mathrm{~h}$ with mouse anti-EnCL3/antiEnCL1/anti-EnCB / control sera diluted 1:100 in PBS-T and then for $1 \mathrm{~h}$ with horseradish peroxidase-labelled goat anti-mouse IgG (Sigma-Aldrich) diluted 1:5000 in PBS-T. Finally, the membranes were developed by the Opti-4CN Substrate Kit (Bio-Rad, Hercules, California).

Purified recombinant proteins $(1 \mu \mathrm{g} /$ well $)$ were resolved by SDS-PAGE in $12 \%$ gels and either stained by CBB or trans-blotted onto a PVDF membrane. His-tagged enzymes were detected on blots either with a mouse biotinylated monoclonal Anti-polyhistidine antibody (Sigma-Aldrich), alternatively with $5 \mathrm{nM}$ iBody4, which is a biotinylated copolymer containing nitrilotriacetic acid-bound nickel cations [46]. In both cases, detection was finalized using peroxidase-labelled streptavidin (Sigma-Aldrich) and the Opti-4CN ${ }^{\text {su }}$ Substrate kit.

\section{Immunohistochemistry}

Deparaffined and rehydrated $5 \mu \mathrm{m}$ sections of worms on slides were heated $3 \times 3 \mathrm{~min}$ in $0.01 \mathrm{M}$ citrate buffer pH 6 containing $0.05 \%$ Tween 20 in a microwave oven $(500 \mathrm{~W})$ and then allowed to cool for $20 \mathrm{~min}$. Subsequently, the sections were blocked and immunostained according to a modified Immunocytochemistry and Immunofluorescence Protocol (Abcam). After an overnight incubation with sera (1:50), the slides were placed in a dark chamber for $1 \mathrm{~h}$ at RT with Goat Anti-Mouse IgG H\&L-Alexa Fluor 568 (Ex: 578 nm/Em: 603 nm, Abcam, Cambridge, United Kingdom) diluted 1:200 in PBS-TX100 with $1 \%$ BSA. Finally, the sections were mounted in Vectashield with DAPI (Vector laboratories, Burlingame, California). Immunofluorescence was observed and photographed under fluorescence or confocal microscope.

\section{Results \\ Bioinformatic and phylogenetic analyses of the primary structures of the enzymes}

Ten unique transcripts encoding different cathepsin L-like sequences were discovered in the transcriptome of adult E. nipponicum. Of the predicted CL amino acid sequences, seven represented complete sequences of mature (processed) enzymes, with six encoding the complete sequences of zymogens including the pro-sequences. Two of the predicted complete zymogen sequences were identical with the sequence of EnCL1 (GenBank: KP793605) and EnCL3 (GenBank: KP793606) obtained previously by reverse transcription of parasite mRNA using degenerate primers, followed by RACE-PCR [12] The complete pro-enzyme molecules were assigned $E n C L 1-E n C L 6$. The remaining four incomplete sequences shared a relatively high similarity with EnCL6: the five close orthologs were therefore assigned EnCL6a-EnCL6e. The alignment of the predicted amino acid sequences of mature EnCLs with cathepsins L1 (GenBank: AAT76664.1) and L3 (GenBank: CAC12807.1) of F. hepatica is presented in Additional file 4, including a mutual comparison of the amino acids of the S2 pocket, which determines the substrate-binding specificity of the enzymes. The alignment of all complete/incomplete amino acid sequences of EnCLs is included in Additional file 5.

Interestingly, EnCL2 displays a substitution of the active site cysteine (Cys25) to serine (Ser25). In terms of amino acid constitutions of the $S 2$ pocket (Additional files 4, 5 and 6), the EnCLs can be divided in 3 groups. One is formed by CL1, CL3, CL4 and CL5, which share the Leu67, Ala133, Leu157 motif (papain numbering used throughout this column), with a slightly hydrophobic Ala being at the bottom of the S2 pocket. The second includes $E n C L 6 a, E n C L 6 b$ and EnCL6e, where Ala133 is replaced by the amphiphilic Gly133 (for EnCL6c and EnCL6d data were not available.). The third group contains only EnCL2, in which the S2 pocket differs substantially from that of the other EnCLs: Ala133 is replaced by the more hydrophobic Val133 at the bottom, and moreover, the two leucines are replaced by Trp67 and Phe157, which could make the $\mathrm{S} 2$ pocket highly hydrophobic. The most variable element in the S2 pocket is the amino acid position 205, where hydrophobic residues occur in CL3, CL4 and CL5, and uncharged hydrophilic residues in CL1 and CL6a. In the case of CL2, residue 205 is a positively charged Arg, whereas in CL6e, it is the negatively charged aspartate.

Some of the EnCLs include potential N-glycosylation sites: CL2 has one and CL4 has two in the mature 
enzyme, others occur in the pro-sequences of CL2, CL4 and CL5. Several O-glycosylation sites have been predicted in all but one $(E n C L 6 b)$ cathepsin $\mathrm{L}$ sequences. Most, however, are located in the pro-domains; CL5 has three, while all the other enzymes except for CL6d have one in the mature part (Table 1, Additional file 5).

The rate of transcription of the particular EnCLs was predicted by a reverse mapping of raw reads towards the selected transcripts. EnCL1 is the most abundant and $E n C L 3$ is the sixth most abundant cathepsin $\mathrm{L}$ in $E$. nipponicum adults (Fig. 1).

Phylogenetic analysis has shown that E. nipponicum cathepsins L separate into two clusters: one contains CL1, CL3 and CL5, and the other includes CL2, CL4 and CL6a. The two clusters belong to separate clades, whereby each of the clusters also includes different cathepsins L of free-living rhabditophorans and CLs of digeneans and cestodes (Fig. 2, Additional file 7). It can thus be concluded that E. nipponicum cathepsins $\mathrm{L}$ are polyphyletic.

The cluster containing $E n C L 1$ and $E n C L 3$, for which the function in blood digestion was confirmed in this study, occurs within the same clade as the CL of another haematophagous monogenean, Protopolystoma xenopodis. The various CLs of the mucophagous Gyrodactylus salaris fall into both clades where the two clusters of EnCLs are located. Interesting is the highly supported position of myxosporean CLs within the neodermatan clade which contains also EnCL2, EnCL4 and EnCL6a.

In stark contrast to the numerous EnCLs, only one transcript encoding $E n C B$ was found in the E. nipponicum transcriptome; it contained the whole sequence of the pro-enzyme. Its closest relative found in the databases is cathepsin B2 of Schistosoma mansoni (GenBank: XP_018651608.1) (see the alignment in Additional file 8).

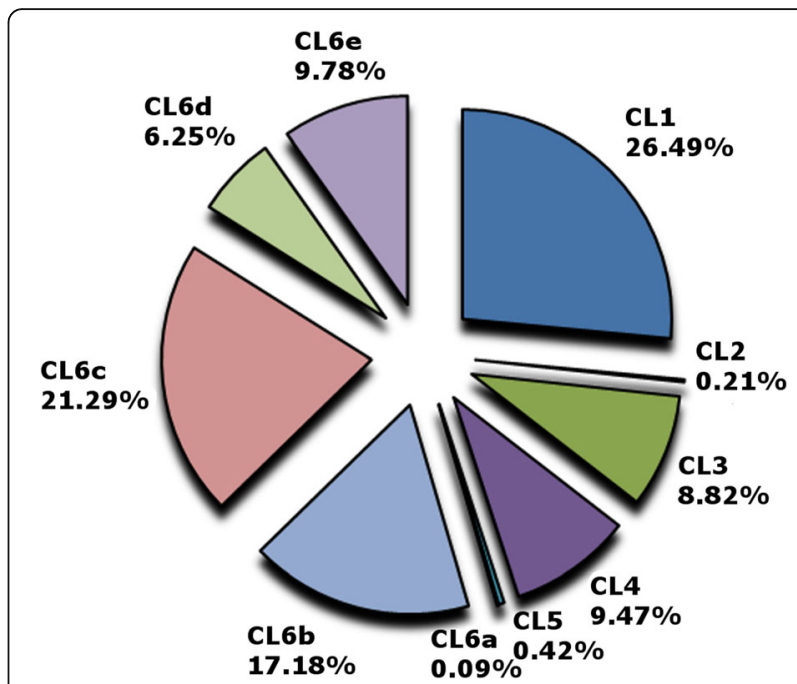

Fig. 1 Relative transcription rate of E. nipponicum cathepsins $L$. The relative transcription rate predicted by a back mapping of raw Illumina reads to the assembled transcripts was calculated as the percentage of all cathepsin L transcripts

Its primary structure contains the typical motif of an occluding loop, which is responsible for peptidyl dipeptidase activity of cathepsins B. One potential $\mathrm{N}$-glycosylation site is positioned on the occluding loop. Seven O-glycosylation motifs were predicted in the molecule, five of them located within the pro-domain. $E n C B$ also contains a modified 'haemoglobinase motif' (213)YWLIANSW-EWGD(226) in the asparagine active site region, which is ascribed to haemoglobinolytic cathepsins B of helminth blood-feeders [47]. The predicted/ computed basic features of all EnCLs and $E n C B$ are summarised in Table 1.

Table 1 Predicted/computed sequence-derived features of $E$. nipponicum cathepsins $L$ and $B$

\begin{tabular}{llllllll}
\hline & Transcript_ID & Bases/AA & Completeness & SP & $\begin{array}{l}\text { pl proenzyme/ } \\
\text { mature enzyme }\end{array}$ & $\begin{array}{l}\text { MW proenzyme/ } \\
\text { mature enzyme (kDa) }\end{array}$ & N-/O-glyc sites \\
\hline CL1 & E_nip_trans_58808_m.372114 & $954 / 317$ & yes & no & $5.86 / 6.08$ & $34.9 / 24.36$ & $0 / 7$ \\
CL2 & E_nip_trans_70234_m.461805 & $1029 / 342$ & yes & yes & $7.23 / 4.87$ & $37 / 24.9$ & $3 / 2$ \\
CL3 & E_nip_trans_02967_m.11341 & $1107 / 368$ & yes & yes & $4.80 / 4.15$ & $38 / 24.1$ & $0 / 5$ \\
CL4 & E_nip_trans_06099_m.25531 & $1062 / 353$ & yes & yes & $5.59 / 4.94$ & $37.6 / 24.2$ & $3 / 8$ \\
CL5 & E_nip_trans_65378_m.396731 & $1071 / 356$ & yes & no & $5.62 / 5.02$ & $39.5 / 24$ & $1 / 8$ \\
CL6a & E_nip_trans_15113_m.115989 & $1059 / 352$ & yes & yes & $5.55 / 4.8$ & $37.7 / 24.4$ & $0 / 2$ \\
CL6b & E_nip_trans_04751_m.20488 & $519 / 173$ & iN & $?$ & $? / ?$ & $? / ?$ & $0 / 0$ \\
CL6c & E_nip_trans_04670_m.20209 & $642 / 214$ & iC & yes & $? / ?$ & $? / ?$ & $0 / 4$ \\
CL6d & E_nip_trans_55822_m.362291 & $705 / 235$ & iC & yes & $? / ?$ & $? / ?$ & $0 / 1$ \\
CL6e & E_nip_trans_60687_m.380367 & $768 / 255$ & iN & $?$ & $? / 4.58$ & $? / 24.47$ & $0 / 2$ \\
CB & E_nip_trans_02724_m.9562 & $1149 / 382$ & yes & yes & $7.21 / 5.55$ & $39.9 / 28.5$ & $1 / 7$ \\
\hline
\end{tabular}




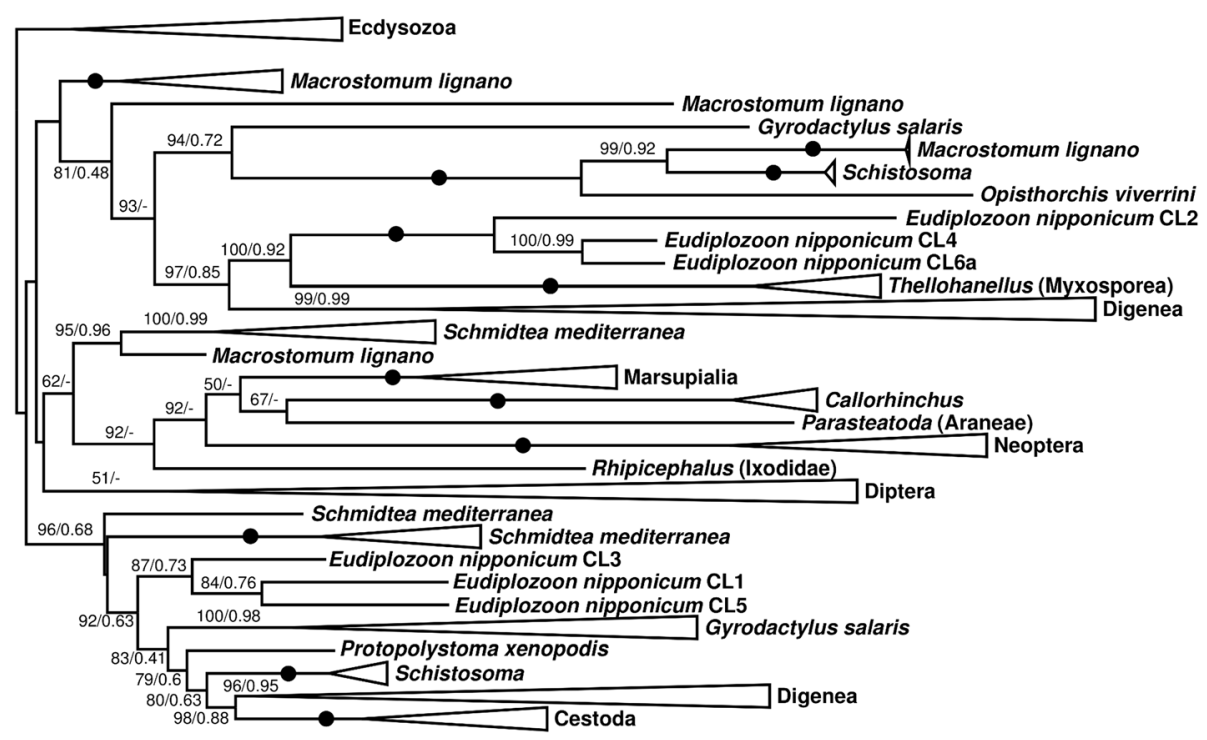

Fig. 2 Collapsed phylogram showing relationships between E. nipponicum cathepsins $L$ and cathepsins $L$ of other selected organisms. Collapsed unrooted maximum-likelihood tree of selected E. nipponicum cathepsins $L$ inferred using the best-fit model $(L G+C 60+G)$. Leaves of related organisms are collapsed. Ultrafast bootstrap supports and posterior probabilities are shown where ultrafast bootstrap is $\geq 50$ or posterior probability $\geq 0$.9. Maximum support (100/1.0) is indicated by a black circle

\section{Functional expression and purification of recombinant cathepsins L1, L3 and B}

$E n C L 1, E n C L 3$ and $E n C B$ were expressed as pro-enzymes in yeasts. Since $y r E n C L 1$ and $y r E n C B$ had undergone self-processing already in yeast cultivation medium, a high activity of both was confirmed in the presence of appropriate fluorogenic peptide substrates. The $y r E n C L 1$ thus migrated in electrophoretic gels as a $\sim 28 \mathrm{kDa}$ dominant double band and reacted on the blot with anti-His-Tag mouse antibody and iBody4 at the same size (Fig. 3). The enzyme was partially purified by Ni-NTA affinity chromatography, but attempts to purify it to homogeneity by ion-exchange chromatography failed due to its poor stability. Activity and inhibition assays were therefore performed with only partially purified enzyme. Additionally,

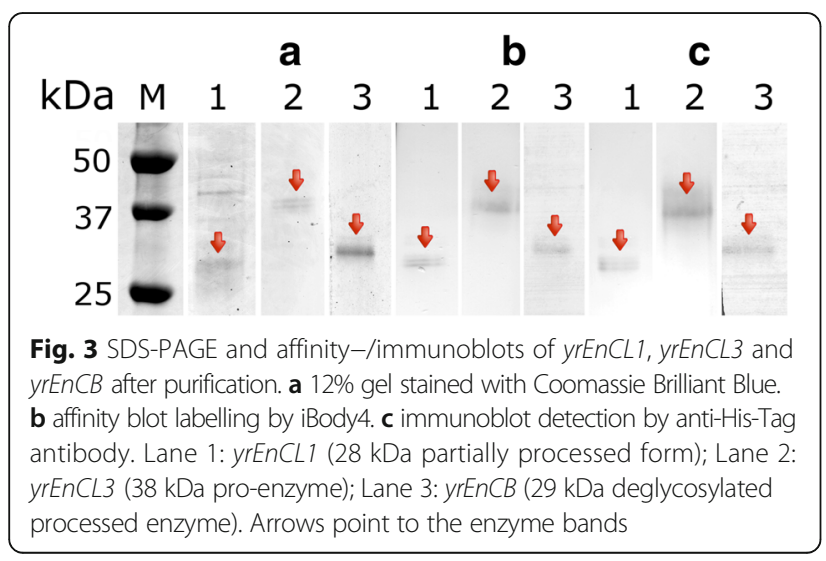

for the production of anti-EnCL1 antibodies it was necessary to use the inactive recombinant form produced in E. coli (brEnCL1).

The affinity purified unprocessed pro-yrEnCL3 migrated in the gel as a double band at $\sim 38 \mathrm{kDa}$, and reacted with the anti-His-Tag antibody and iBody4 at the same size (Fig. 3). Autocatalytic processing of pro-yrEnCL3 was most effective at $\mathrm{pH} 4$, and resulted in a $\sim 28 \mathrm{kDa}$ band in the gel. Processing of the zymogen was less effective at $\mathrm{pH} 5$, while at $\mathrm{pH} 6$ it did not take place even after prolonged incubation. Affinity probe BODIPYgreen-DCG-04 bound strictly to the mature enzyme, while in the proenzyme no labelling was observed (Fig. 4). Edman degradation revealed LPTDVD sequence at the N-terminus of the processed EnCL3. Since the processed enzyme had limited stability, the purified pro-enzyme was used for the immunisation of mice.

$Y r E n C B$ pre-purified on a Ni-NTA column migrated as a broad band around $50 \mathrm{kDa}$. After enzymatic deglycosylation and subsequent purification by ion-exchange chromatography, it migrated as a $\sim 29 \mathrm{kDa}$ band in the gels, which correlated with the results of Western and affinity blotting (anti-His-Tag antibody, iBody4) (Fig. 3).

\section{Activity and inhibition assays}

Both EnCLs efficiently hydrolysed the FR substrate, and to a lesser extent the LR. No activity towards RR, PR and GPR was detected. The optima for hydrolysis of both substrates were $\mathrm{pH} 5.5$ for $y r E n C L 1$ and $\mathrm{pH} 6$ for $y r E n C L 3$. The preferred substrate for $y r E n C B$ was the 


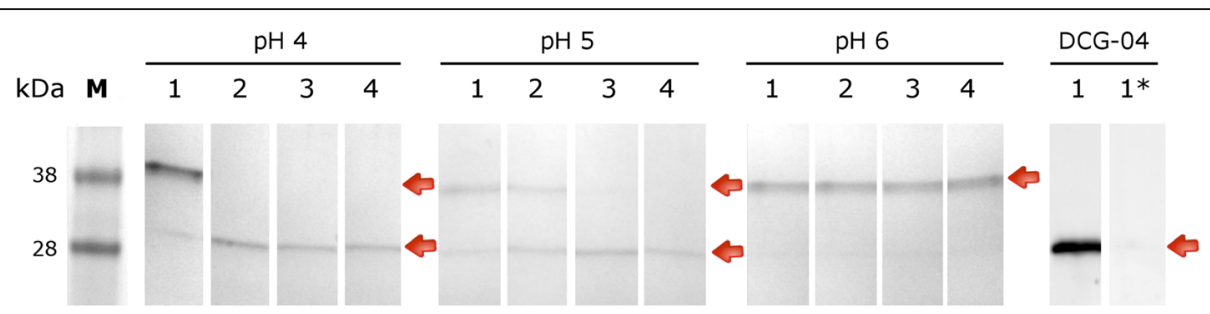

Fig. 4 SDS-PAGE and active site labelling of purified pro-yrEnCL3 after auto-activation at various pH. The $38 \mathrm{kDa}$ zymogen was autocatalytically processed to a $28 \mathrm{kDa}$ form during incubation in $50 \mathrm{mM} / 100 \mathrm{mM} \mathrm{CPB}$ containing $2 \mathrm{mM}$ DTT at $37^{\circ} \mathrm{C}$. After incubation at various pH values, the protein was run in 12\% polyacrylamide gels and stained with Coomassie Brilliant Blue (pH 4, pH 5 and pH 6). Lane 1: 15 min incubation; Lane 2: 30 min; Lane 3: 60 min; Lane 4120 min. DCG-04, a 12\% gel showing active site labelling of the processed enzyme with fluorescent affinity probe BODIPY green DCG-04. Lane 1: labelling by the affinity probe after 30 min incubation at $\mathrm{pH}$; $\mathbf{1}^{*}$, binding of the affinity probe was blocked by E-64 inhibitor. The $38 \mathrm{kDa}$ zymogen and the $28 \mathrm{kDa}$ processed enzyme are indicated by arrows

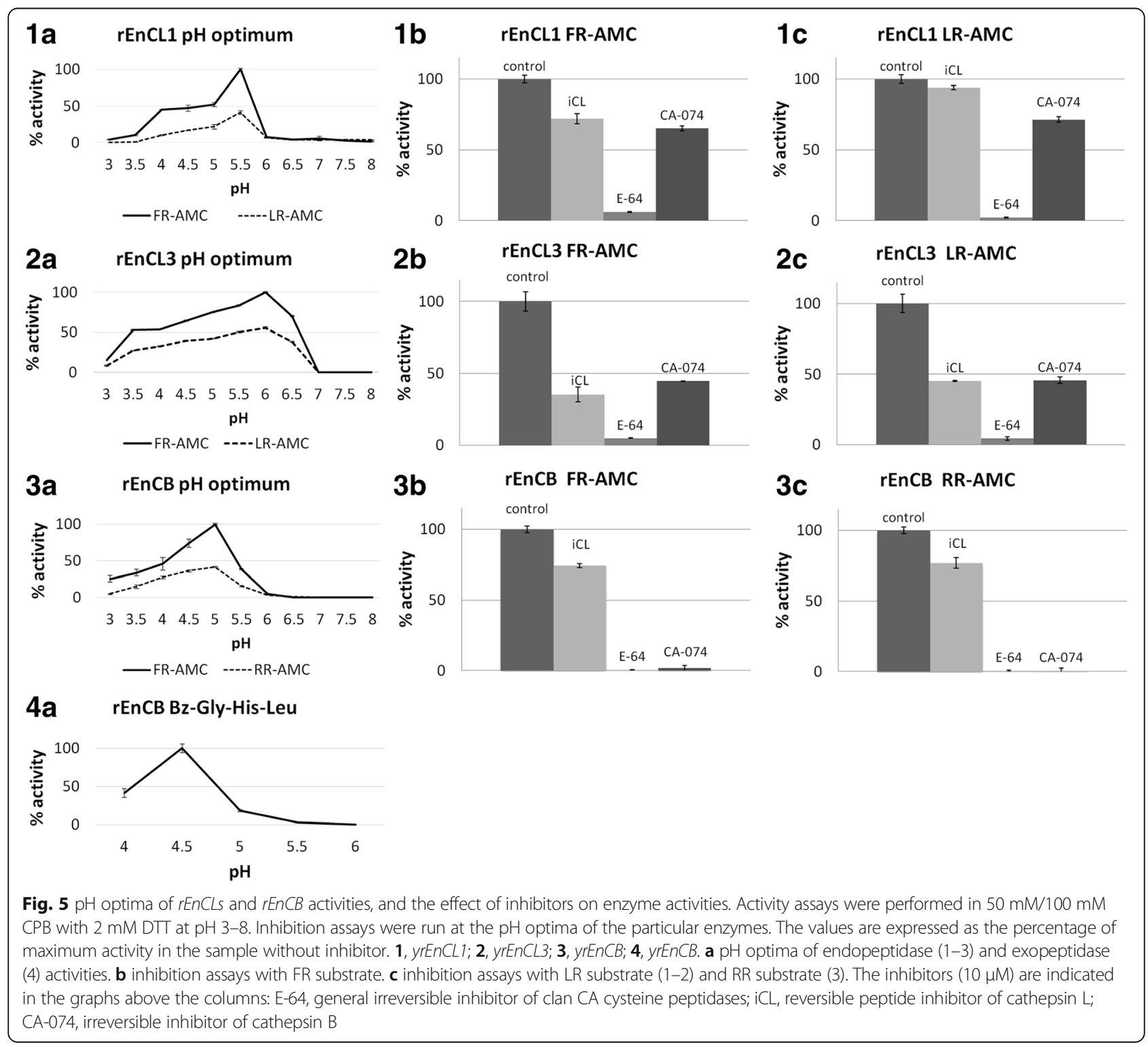


FR, while RR was cleaved less efficiently at the optimum of pH 5 (Fig. 5).

Activity of all three enzymes was effectively inhibited by $10 \mu \mathrm{M}$ E-64 (the general inhibitor of clan CA family C1 cysteine peptidases) in the presence of either substrate. The cathepsin B-selective inhibitor CA-074 had completely suppressed the activity of $y r E n C B$ at $10 \mu \mathrm{M}$ and diminished the activity of $y r E n C L 1$ and $y r E n C L 3$ to $\sim 70 \%$ and $45-65 \%$, respectively, depending on the substrate. The reversible peptide inhibitor of cathepsin $\mathrm{L}(10 \mu \mathrm{M}, \mathrm{iCL})$ partially inhibited all three cathepsins but was most effective in the case of $y r E n C L 3$ with both substrates (c.40\% remaining activity). The inhibition rate of $y r E n C L 1$ was comparable with the effect on $y r E n C B$ (c.90-70\% remaining activity) (Fig. 5).

The exopeptidase (dipeptidyl peptidase) activity of $y r E n C B$ peaked at $\mathrm{pH} 4.5$, then rapidly dropping to $c .40 \%$ at $\mathrm{pH} 4$, and to less than $20 \%$ at pH 5 (Fig. 5).

\section{Hydrolysis of macromolecular substrates by recombinant cathepsins $L$ and $B$}

Both $E n C L s$ and $E n C B$ efficiently degraded selected protein substrates (bovine haemoglobin and albumin, human collagen type I and fibrinogen) at $\mathrm{pH}$ 4.5. EnCLs readily hydrolysed also human IgG, which was poorly cleaved by $y r E n C B$ at $\mathrm{pH}$ range $4.5-6.0$ (Fig. 6). While the action of $y r E n C L 1$ did not vary substantially between $\mathrm{pH} 4.5-6.0$, $y r E n C L 3$ did not act on the substrates (except fibrinogen) at $\mathrm{pH} 5.0$ or higher (not shown). At $\mathrm{pH}$, hydrolysis of all the macromolecular substrates by $y r E n C B$ failed (not shown).

\section{Western blotting and immunohistochemistry}

Specificity of mouse anti-EnCL1, anti-EnCL3, and anti$E n C B$ antibodies was verified on immunoblots of the soluble worm extracts. The detected bands appeared at $\sim 24 \mathrm{kDa}$ (EnCL1), $\sim 28 \mathrm{kDa}(E n C L 3)$ and $\sim 29 \mathrm{kDa}$ $(E n C B)$. Reactions of the particular sera with the appropriate enzyme bands were highly specific (Fig. 7), so they could be used for immunohistochemistry. Control pre-immune sera did not exhibit any reaction.

The reactions of specific antibodies with histological sections of the worms showed the presence of both $E n C L 1$ and EnCL3 in the lumen of the gut (Fig. 8). No reaction was observed in the haematin digestive cells or outside the digestive system.

Anti-yrEnCB antibodies reacted with vesicular structures in the vitelline cells of the adult parasites (Fig. 8). All control sera displayed no reaction.

\section{RNA in situ hybridisation}

In situ hybridisation with specific antisense-RNA probes showed a localisation of EnCL1 and EnCL3 transcripts specifically in the haematin (digestive) cells of the worms' gastrodermis (Fig. 9). No reactivity was found in other parts of the parasite body, including tegument, vitelline cells and the initial part of the digestive tract. The same pattern of reactivity was obtained when the sense-RNA probes were used. This was not a non-specific reaction, as demonstrated by the strand-specific RT-PCR which confirmed the presence of anti-sense transcripts in $E$. nipponicum. In the negative controls (without any probe), no reaction was observed.

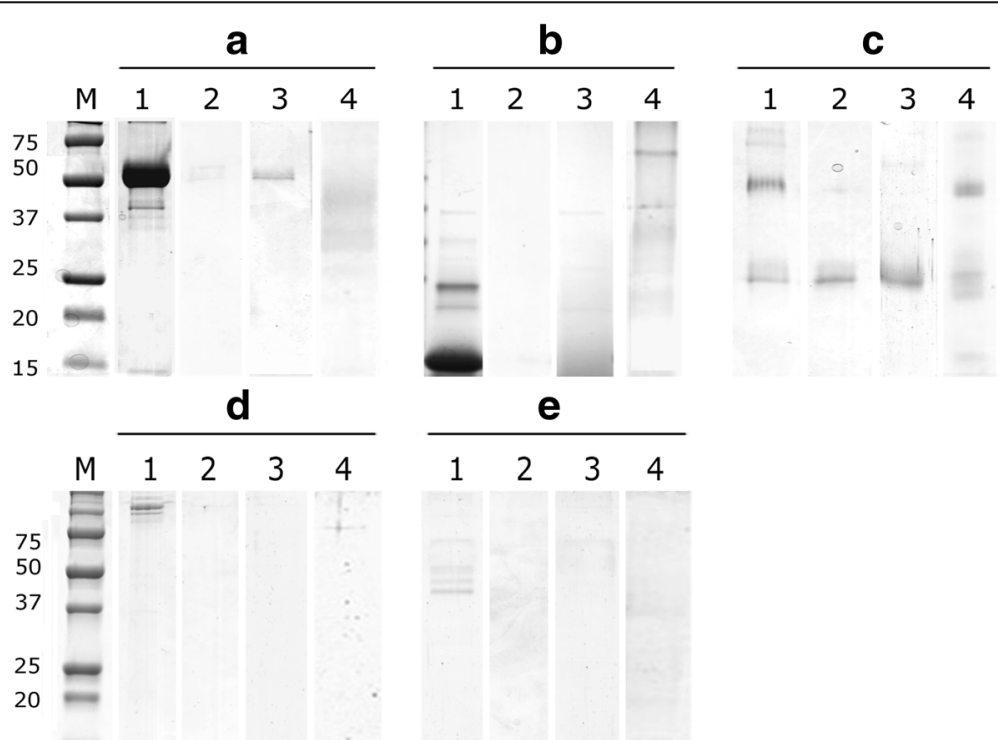

Fig. 6 SDS-PAGE of protein substrates degraded by recombinant EnCL1, EnCL3 and EnCB. Prior to electrophoresis in 12\% polyacrylamide gel, the enzymes were incubated overnight with various protein substrates in $50 \mathrm{mM} / 100 \mathrm{mM} \mathrm{CPB} \mathrm{pH} 4.5$ containing $2 \mathrm{mM}$ DTT. a Bovine serum albumin. b Bovine haemoglobin. c Human lgG. d Human type I collagen. e Human fibrinogen. Lane 1: controls (substrates without enzyme); Lane 2: yrEnCL1; Lane 3: yrEnCL3; 4: yrEnCB. Gels were stained with Coomassie Brilliant Blue 


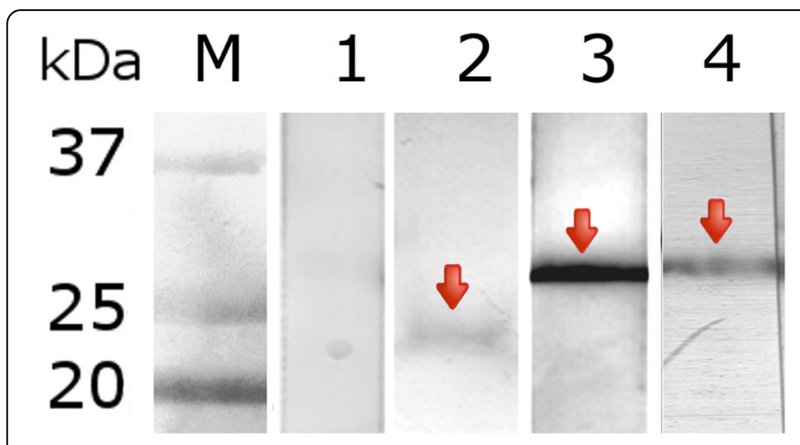

Fig. 7 Reactions of anti-cathepsin antibodies with proteins on blots of E. nipponicum soluble extracts. Lane 1: mixed control (pre-immune sera); Lane 2: anti-brEnCL1 antibodies; Lane 3: anti-pro-yrEnCL3; Lane 4: anti-yrEnCB. Arrows point to the enzymes detected by specific antibodies

\section{Discussion}

In a previous study [12], we proposed that cathepsin L-like peptidases, in cooperation with cathepsins D and B, play a leading role in the digestion of haemoglobin in the sanguinivorous monogenean E. nipponicum. Although the presence of cathepsins $\mathrm{L}$ in soluble extracts of the worms was suggested by several methods in that study, the large number of different cathepsin L transcripts (10 in total) found in the transcriptome of the adult life stage was not anticipated. In other blood-feeding helminths, such as Fasciola hepatica, multiple cathepsins L are involved in feeding and other important tasks, such as tissue migration, immune evasion, and possibly egg production and metacercarial excystment $[48,49]$. Small changes (even single amino acid substitutions) in the primary structure, but especially in the S2 subsite of the active site of those cathepsin L types/isoforms, alter the substrate specificity [50-52]. The production of peptidases with overlapping

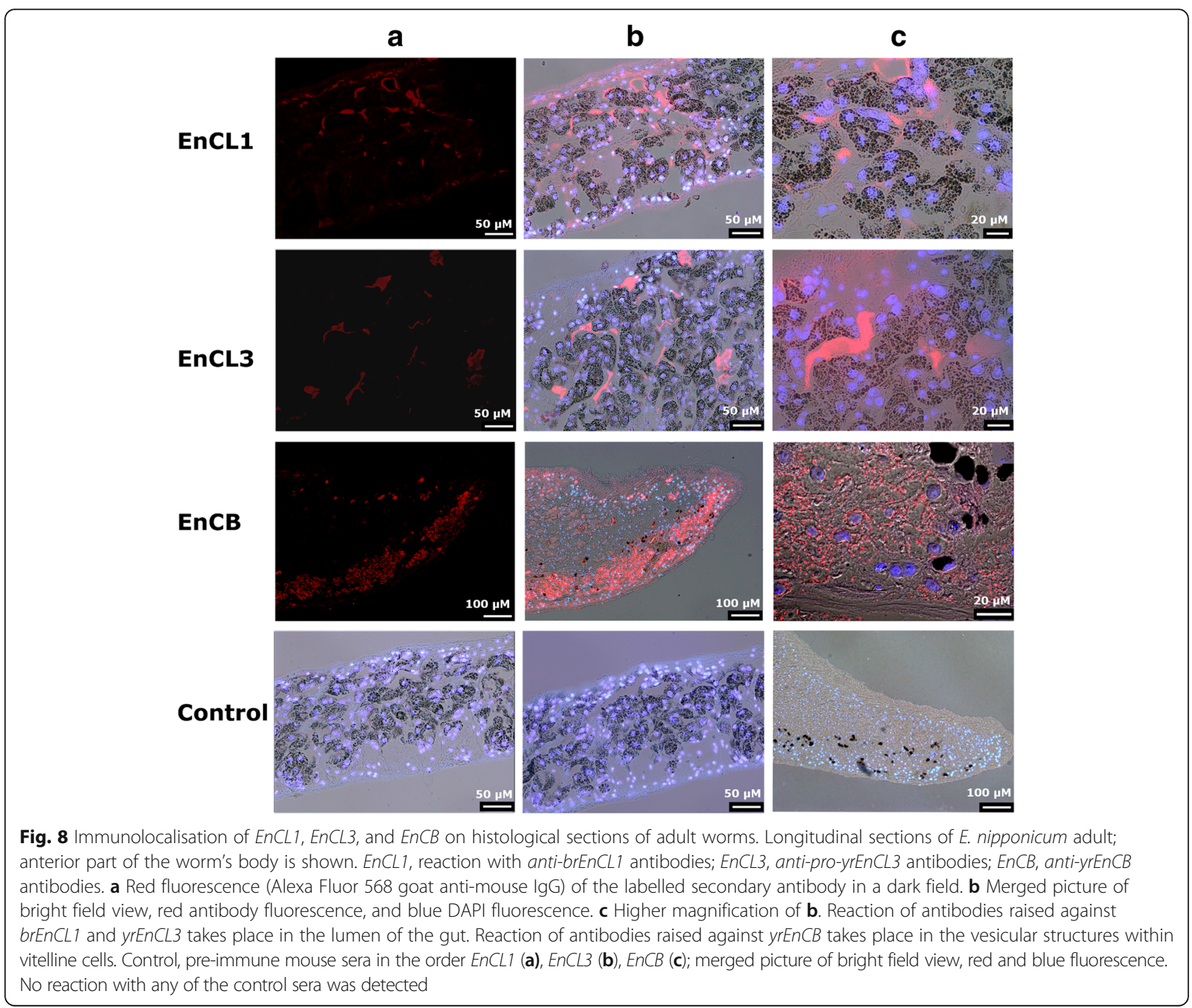




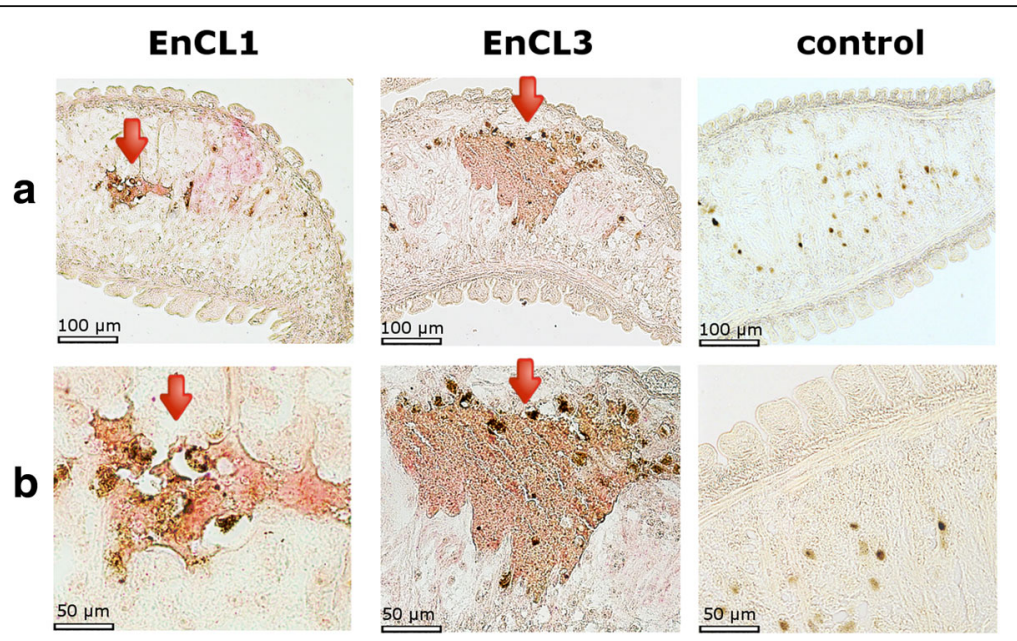

Fig. 9 Localisation of mRNA encoding EnCL1 and EnCL3 using RNA in situ hybridisation. Longitudinal sections of E. nipponicum adult worms incubated with specific EnCL1/EnCL3 antisense-RNA probes. a Reaction in haematin cells of the digestive tract. b Higher magnification of the reacting areas from the same section. Controls were performed without any probe because the worms also produce anti-sense transcripts

specificities enables the parasite to degrade host-derived macromolecules more efficiently.

All but one of the EnCLs characterised in this study possess a typical catalytic triad of cysteine peptidase active site (Cys/His/Asn); the exception is EnCL2, in which the active site cysteine (Cys25) is replaced by serine (Ser25). However, as described for congopain (cathepsin L-like peptidase) in Trypanosoma congolense, Cys-to-Ser mutation need not necessarily affect the proteolytic ability of an enzyme [53]. It has been also suggested that Cys-to-Ser mutation in the cysteine peptidases of parasites might be a way to moderate oxidation of the active site in case parasites have to deal with an oxygenrich environment, such as blood or external environment [17]. In addition to this substitution in the active site, EnCL2 has in its S2 pocket Trp at position 67 (papain numbering used throughout the Discussion), which makes it a potential collagenase/elastase. Presence of the bulky aromatic residues Tyr67/Trp67 is typical of $F$. hepatica cathepsins L2 and L3, and of human cathepsin K, which all efficiently cleave collagen and elastin of the connective tissues due to their ability to accommodate Pro residues at P2 position in the substrates via a stabilising interaction with Tyr67/Trp67 [50-52, 54, 55]. However, the functionality and possible role of EnCL2 has not yet been studied. Its expression rate in adult worms is very low, which indicates that the enzyme is predominantly employed by another life stage (the larva: oncomiracidium; or the postoncomiracidial stage: diporpa), or in some less prominent processes unconnected with blood digestion.

Since the composition of the S2 pocket is the key determinant of substrate specificity of papain-like cysteine peptidases [56], the amino acid sequences of EnCLs were aligned (Additional files 4, 5 and 6) and their S2 residues (positions 67, 133, 157, 205) compared. The comparison of various EnCLs revealed the existence of three groups differing in the polarity and charge of the $S 2$ pocket. In $E n C L 2$, the $\mathrm{S} 2$ pocket is strongly hydrophobic and carries a positive charge. EnCL1, EnCL3, EnCL4 and EnCL5 have a moderately hydrophobic S2 pocket with a neutral charge. The least hydrophobic S2 pocket is found in the closely related EnCL6 orthologs; it has a neutral charge in EnCL6a and 6b, and a negative charge in EnCL6e (for $E n C L 6 c$ and $6 \mathrm{~d}$ the complete data are not available). The presence of a negatively charged Asp205 in the S2 pocket may endow EnCL6e with the ability to accommodate a positively charged Arg at P2 position of the substrate, which would give this cathepsin $\mathrm{L}$ ortholog a cathepsin B-like substrate specificity. Additionally, the group of $E n C L 6$ orthologs is atypical among cathepsins $\mathrm{L}$ by having an amphipathic Gly133 in the S2 pocket. This feature is, however, typical of cathepsins S, and Gly133 can also be found in a few other, yet unclassified, clan CA family C1 peptidases, such as the plerocercoid growth factor of the larval stage of the tapeworm Spirometra (Genbank: BAB62718.1) (see the alignment in Additional file 6). Nonetheless, specificity of the S2 pocket in papain-like peptidases is largely determined by the residue 205 . Cathepsins $\mathrm{S}$ contain at this position a large aromatic non-polar Phe, which implies a short and more restricted pocket. Cathepsins $\mathrm{L}$, on the other hand, usually include at this position branched aliphatic residues, which enables the accommodation of larger aromatic residues [57]. This was not observed in EnCL6a, which contains a polar Ser205. It thus seems that diversity in the composition of $\mathrm{S} 2$ subsites of E. nipponicum cathepsins L gives the parasite a wide array of efficient proteolytic tools with diverse affinities to potential P2 positions of protein substrates. 
Phylogenetic analysis seems to support a supposition, inferred from the structural data and relative rates of expression, that in E. nipponicum, cathepsins L may be involved in various biological processes. The two functionally characterised enzymes, EnCL1 and EnCL3, which seem to play a central role in blood digestion by the parasite, form a monophyletic group with EnCL5. The other monophyletic clade formed by EnCL2, $E n C L 4$ and EnCL6 is relatively distant from the first group, thus suggesting involvement in other processes or, in the case of the least proportionally represented transcripts such as EnCL2 and EnCL6a, a more substantial expression of these orthologs in other life stages. The presence of myxosporean-like cathepsins L within the otherwise purely neodermatan clade may lead to a speculation about a lateral gene transfer, since myxosporeans share their fish hosts with monogeneans. Investigation of this possibility would, however, require a thorough analysis that is beyond the scope of the present study. Moreover, it should also be noted that the evolution of genes need not follow the branching structure of species-level evolutionary trees due to mechanisms such as gene duplication and independent gene loss. Particularly, genes of parasites are prone to phylogenetic artefacts caused by attraction between long branches, an effect we tried to mitigate by using site-heterogeneous mixture models [58].

Surprisingly, only one transcript coding for cathepsin B was found in the transcriptome of adult $E$. nipponicum. Amino acid sequence of $E n C B$ had shown that this enzyme is a typical representative of cathepsins $B$, including its active site, composition of the S2 subsite, and the occluding loop responsible for exopeptidase activity. It also contains a 'haemoglobinase motif' assigned to helminth cathepsins B with substrate specificity for haemoglobin [47]. In the case of $E n C B$, this motif is slightly modified in its $C$-terminal region, where the original sequence described for helminth haemoglobinases 'DWGE' is replaced by 'EWGD' (Additional file 8). Nevertheless, as we experimentally verified, these purely conservative amino acid substitutions did not affect the ability of $E n C B$ to cleave haemoglobin. Similar substitutions in the motif were described for schistosome cathepsins B2 (S. mansoni and Trichobilharzia regenti) that were found only outside the parasites' digestive system; it is assumed that their functions do not include the digestion of haemoglobin $[59,60]$. In contrast to $E n C B, T$. regenti CB2 degraded haemoglobin at a negligible rate.

In the process of heterologous functional expression in P. pastoris, $\mathrm{YrEnCL1}$ had undergone auto-activation in the cultivation medium by cleaving-off a significant proportion of its pro-sequence and migrated on SDS-PAGE as a $28 \mathrm{kDa}$ band (the value thus differed from the expected $\sim 35 \mathrm{kDa}$ and $\sim 24 \mathrm{kDa}$ of the zymogen and fully processed enzyme, respectively). Other, less abundant bands also occasionally appeared in the gel; mass spectrometry had confirmed they were fragments of $y r E n C L 1$, generated probably by auto-degradation (data not shown). This is consistent with the very limited stability of the partially processed enzyme observed during purification. Monospecific anti-yrEnCL1 antibodies did, however, react with a $\sim 24 \mathrm{kDa}$ band on blots of soluble worm extracts, indicating a full processing of EnCL1 under natural conditions. The $y r E n C L 3$, on the other hand, was obtained as a relatively stable zymogen. It was capable of autocatalytic activation under acidic conditions, but the size of the resulting product, $\sim 28 \mathrm{kDa}$, was also somewhat higher than the predicted mass of $\sim 24.1 \mathrm{kDa}$ of the mature enzyme. Nevertheless, Edman degradation confirmed an amino acid sequence at the $\mathrm{N}$-terminus of the processed yrENCL3 which had been predicted by bioinformatical methods, thus indicating that the processing was complete. Monospecific anti-yrEnCL3 antibodies reacted similarly with a $\sim 28 \mathrm{kDa}$ band on blots of soluble worm extracts. Given that the EnCL3 does not possess any N-glycosylation motifs, these differences cannot be explained by either natural glycosylation of the enzyme or by $\mathrm{N}$ hyperglycosylation from $P$. pastoris in the case of the recombinant enzyme. It could be speculated that the observed shift in $\mathrm{Mw}$ of the enzyme was caused by disruption of disulphide bonds stabilizing cathepsin L structure, which resulted in mobility change during electrophoresis [60, 61]. Although EnCL3 possesses one potential $\mathrm{O}$-glycosylation site in the mature part, the prediction software on the NetOglyc server produces predictions of mucin type GalNAc O-glycosylation sites in mammalian proteins, and the situation may be different in invertebrates. Moreover, we are not aware of any descriptions in literature of a cathepsin L with experimentally confirmed O-glycosylation.

S2 subsite specificity of $y r E n C L 1$ and $y r E n C L 3$ was tested over a range of $\mathrm{pH}$ with a set of oligopeptide synthetic substrates, varying in their Phe, Leu, Arg, and Pro at P2 position. As predicted from the composition of the $\mathrm{S} 2$ pockets, the enzymes were not able to cleave substrates containing Pro or Arg in this position. Consistently with most papain-like peptidases, both enzymes preferred at this position the bulky hydrophobic aromatic Phe and the branched aliphatic Leu, in order Phe $>$ Leu [56]. The enzymes did, however, differ in their $\mathrm{pH}$ profiles of activity towards substrates, so that whereas $y r E n C L 1$ was active only in a narrow $\mathrm{pH}$ range with a $\mathrm{pH}$ optimum at 5.5, $y r E n C L 3$ had a broader $\mathrm{pH}$ activity profile with an optimum at $\mathrm{pH} 6$. The slightly acidic $\mathrm{pH}$ optima correspond to those of cathepsin L-like peptidases of other blood-feeding parasites [61-64].

As expected, activity of both of the recombinant EnCLs was inhibited by the general papain-like cysteine 
peptidase inhibitor E-64. Surprisingly, however, the iCL, a highly potent amphiphilic reversible peptide inhibitor of human cathepsin L [65], had but a little effect on the enzymes' activity, although it has Leu at the P2 position. One can thus hypothesise that a lower affinity to Trp at P1 position, found also in, e.g. cathepsin L of Leishmania mexicana [56], might account for this result. Remarkably, CA-074 (a specific inhibitor of cathepsin B) also partially reduced the activity of both cathepsins L. It has been reported elsewhere [66, 67], however, that CA-074 can decrease the activity of cathepsins $\mathrm{L}$ under reducing conditions and that could also explains our results.

Activity profiles, $\mathrm{pH}$ optimum, specificity to peptide substrates, inhibition by CA-074 and exopeptidase activity clearly show that $E n C B$ is a typical member of cathepsins $B$ group. The pro-enzyme had undergone auto-activation either in the yeast medium or during purification, and enzymatic deglycosylation demonstrated hyperglycosylation of the recombinant enzyme by yeast, which corresponds to the presence of an $\mathrm{N}$-glycosylation motif in the $E n C B$ molecule. Anti-yrEnCB monospecific antibodies detected native $E n C B$ on blots of soluble worm extracts at $\sim 29 \mathrm{kDa}$, which is adequate to the expected size of the processed enzyme which was probably slightly glycosylated naturally.

Both recombinant EnCL1 and EnCL3 efficiently degraded various blood proteins and type I collagen of the connective tissue. Unlike synthetic peptide substrates, macromolecular substrates (except for fibrinogen) were cleaved only at $\mathrm{pH} 4.5$ by $y r E n C L 3$, while $y r E n C L 1$ hydrolysed all macromolecular substrates at $\mathrm{pH}$ 4.5-6.0. Activity at low $\mathrm{pH}$ values is in accordance with the site of expression of both genes encoding the enzymes that was localised by in situ hybridisation in the haematin (digestive) cells, and with the presumed intracellular digestion of haemoglobin in the endolysosomal vesicles where $\mathrm{pH}$ below 4.5 would be expected [68]. Both enzymes, however, were also immunolocalised in the lumen of the parasite's gut. For other blood-feeding helminths, it has been estimated that the $\mathrm{pH}$ in the gut lumen is only slightly acidic (c.5.5-6.5) $[43,69,70]$, and digestion therefore takes place in a local microenvironment between the lamellae of the digestive cells where the $\mathrm{pH}$ is more acidic ( 4.5), as documented for F. hepatica [71]. Haematin cells of another studied blood-feeding diplozoid monogenean, Paradiplozoon homoion, also contain numerous lamellae on the side facing the lumen of the gut. Host erythrocytes are lysed in proximity of the haematin cells and released haemoglobin is taken in by lamellae of the cells via pinocytosis [9]. These facts, together with presence of EnCL1 and EnCL3 in excretory/secretory products of the worms [12], and with the results of immunolocalisation, imply that in diplozoid monogeneans extracellular, luminal digestion of blood can take place. Moreover, if the enzymes are released from the gut lumen into host circulation, they could potentially participate in some host-parasite interactions by affecting, e.g. fibrinolysis (they cleaved fibrinogen in vitro) or immune response (degradation of immunoglobulins).

Surprisingly, both EnCLs hydrolysed collagen type I. This ability was attributed in human body primarily to family $\mathrm{C} 1$ lysosomal cysteine peptidase cathepsin $\mathrm{K}$. Because of acceptance of Pro in the P2 position, cathepsin $\mathrm{K}$ can completely degrade collagen by cleaving within the repeated Gly-Pro-Xaa motifs that occur in the triple helix of collagen [54]. The ability to accommodate substrates with P2 proline has also been observed in some cathepsins $\mathrm{L}$ of F. hepatica, namely FhCL2, FhCL3 and FhCL5 [50, 55, 72]. On the other hand, neither $y r E n C L 1$ nor $y r E n C L 3$ cleaved synthetic substrates GPR and PR. So, though both enzymes degraded native collagen, they probably do not possess intrahelical activity and this likely makes them less effective collagenases. Such a phenomenon has been described in human cathepsin $\mathrm{L}$ and FhCL1 [51, 54].

During the localisation of expression of EnCL1 and EnCL3 by RNA in situ hybridisation, sense-RNA probes used as controls had shown similar reaction as antisense probes. It is known from the literature that over $30 \%$ of genes transcribed in humans also have antisense transcription [73], and antisense transcripts have been described for genes in a diverse group of eukaryotes [74-76]. The use of a specific strand RT-PCR has revealed that the $E n C L 1$ and EnCL3 encoding genes also have naturally present antisense transcripts. Since the cathepsin L-like peptidases play important roles during the life of the monogeneans, antisense transcripts may be associated with the regulation of gene transcription [77].

It has originally been thought that $E n C B$ is also involved in the proteolytic cascade of blood digestion in E. nipponicum [12]. In haematophagous parasites, many cathepsins $\mathrm{B}$, together with other peptidases of the digestive cascades, function as important enzymes with a mixed mode of action, as endopeptidases that cleave proteins into smaller fragments and as exopeptidases (peptidyldipeptidases, carboxypeptidases) that remove dipeptides from the C-termini of those protein fragments due to the presence of the occluding loop [78]. Immunofluorescence with monospecific anti-yrEnCB antibodies clearly showed the localisation of $E n C B$ in vesicular structures in vitelline cells, but its presence in the digestive tract has not been detected at all. We could thus speculate on its function in vitellogenesis. The presence of cysteine peptidases in the reproductive system has been detected also in other helminths [79-82], but their role in worms' reproduction is still speculative. The lack of exopeptidase activity in the gut due to the absence of cathepsin B may be compensated by the activity of cathepsins $\mathrm{C}$; at least three different transcripts of these 
dipeptidyl peptidases (aminopeptidases) were found in E. nipponicum transcriptome (not shown).

\section{Conclusions}

To our knowledge, this work represents the first comprehensive exploration and functional annotation of a group of peptidases from a monogenean transcriptome. It also describes the very first functional characterisation of recombinant cysteine peptidases of monogenean origin. The lack of experimental data from other monogeneans, particularly those of a high economic importance (such as Gyrodactylus salaris, Benedenia spp.), unfortunately precludes the possibility of drawing any comparisons. We have shown that the haematophagous monogenean E. nipponicum possesses a wide range of endopeptidases of cathepsin L type, most of which are highly expressed in adult worms. Some of these enzymes possess structural features rather unusual in cathepsins L. It holds true especially for the composition of S2 subsite of the active site, which is among the studied enzymes rather variable and includes even substitutions typical more of cathepsins B and cathepsins S. This expands our understanding of the structural diversity of cathepsins $\mathrm{L}$ in general. In contrast to the rich world of cathepsins $\mathrm{L}$, the presence of just one cathepsin B in the worms was somewhat surprising. Despite its ability to hydrolyse haemoglobin in vitro, $E n C B$, due to its localisation in the vitelline cells of the parasite, does not seem to be involved in digestion. On the other hand, functional characterisation and localisation has revealed that $E n C L 1$ and $E n C L 3$ are important in the digestion of blood. Despite previous claims, it seems that the digestive process takes place not only within the intracellular lysosomal cycle, but at least in part also outside the gastrodermis, in the lumen of the gut. From there, the EnCLs may be eventually released into the blood circulation of the host and participate in elicitation of immune response and/or immunomodulation. Genomic data, currently available only for the mucophagous polyonchoinean G. salaris and the haematophagous heteronchoinean Protopolystoma xenopodis, and transcriptomic data from the mucophagous polyonchoinean Neobenedenia melleni, open a possibility for future comparative biochemical research of the two trophic strategies characteristic of the monogeneans. For example, preliminary data explorations show that mucus feeders may employ elastase-like serine peptidases that have not been found in blood-feeders (Mikeš, unpublished). Further research is likely to provide data interesting from an evolutionary point of view and information essential to understanding parasite-host interactions on a molecular level in this group of parasites, which would be of interest especially in context of pathogenesis and fish immunity. Although parasite peptidases have often been seen as promising targets for the development of vaccines or new inhibitor-based remedies, in the case of monogeneans we are somewhat sceptical regarding this possibility. The main reasons why we do not believe this is a promising direction are the likely costs linked to a complicated handling of large numbers of individual fish hosts in aquaculture, difficulties with administering a correct dosage of medication (peptidase inhibitors) in an aquatic environment (assuming that a peroral formula would even be available) and the necessity of repeated administering in order to reach a lethal effect on the parasites. At the moment, most methods applied against monogeneans rely on mass treatment of farmed fish by readily available, low-cost chemical biocides, such as hydrogen peroxide, or antihelminthics, such as praziquantel [83].

\section{Additional files}

Additional file 1: Primers for the expression of yrEnCL1, yrEnCL3, and yrEnCB in P. pastoris. (PDF $239 \mathrm{~kb}$ )

Additional file 2: Primers for the expression of brEnCL 1 in E. coli. (PDF $83 \mathrm{~kb}$ )

Additional file 3: Specific EnCL1/EnCL3 primers for the synthesis of probes for RNA in situ hybridisation. (PDF $84 \mathrm{~kb}$ )

Additional file 4: Amino acid sequence alignment of E. nipponicum cathepsins $L$ with cathepsins $L$ of $F$. hepatica. Signal peptides and pro-sequences were omitted. Numbers at the end of the lines show amino acid numbering of particular mature parts of the enzymes. Catalytic triad of the active site $(\mathrm{C}, \mathrm{H}, \mathrm{N})$ is marked by triangles. Conserved motifs around active site residues are shaded in grey. Residues within the S2 subsite of the active site involved in determining the substrate specificity are shaded in black and indicated with numbers (papain numbering). Tripeptides of potential N-glycosylation sites are boxed. Predicted O-glycosylated residues are marked by grey squares. The alignment was made using the sequences of $F$. hepatica mature cathepsins L1 (GenBank: AAT76664.1) and L3 (GenBank: CAC12807.1). (PDF 1949 kb)

Additional file 5: Multiple alignment of all the complete/incomplete amino acid sequences of E. nipponicum cathepsins $L$ inferred from the transcriptome of adult worms. Signal peptides are shaded in dark grey, position of the pro-region cleavage site is marked by arrows. ERFNIN- and GNFD-like motifs are underlined and indicated by underlined headings. The catalytic triad of the active site $(\mathrm{C}, \mathrm{H}, \mathrm{N})$ is marked by triangles. Conserved motifs around active site residues are shaded in light grey. Residues within the $\$ 2$ subsite of the active site involved in determining the substrate specificity are shaded in black and indicated with numbers (papain numbering). Tripeptides of potential N-glycosylation sites are boxed. Predicted O-glycosylated residues are marked by grey squares. (PDF 5783 kb)

Additional file 6: Composition of S2 subsites of the active sites shown in the alignment of parts of amino acid sequences of E. nipponicum cathepsin L orthologs $6 \mathrm{a}, 6 \mathrm{~b}$ and $6 \mathrm{e}$ with human cathepsin $S$ and with plerocercoid growth factor of Spirometra tapeworm. The catalytic triad of the active site $(\mathrm{C}, \mathrm{H}, \mathrm{N})$ is marked by triangles. Conserved motifs around active site residues are shaded in grey. Residues within the S2 subsite of the active site involved in determining the substrate specificity are shaded in black and indicated with numbers (papain numbering). Abbreviations: PGF, Spirometra erinaceieuropaei plerocercoid growth factor (Genbank: BAB62718.1); HsCS, human cathepsin S (Genbank: AAC37592). (PDF $1406 \mathrm{~kb}$ )

Additional file 7: Phylogram showing relationships between $E$. nipponicum cathepsins $L$ and cathepsins $L$ of other selected organisms. Unrooted maximum-likelihood tree of selected E. nipponicum cathepsins $L$ inferred using the best-fit model $(L G+C 60+G)$. Ultrafast bootstrap supports and posterior probabilities are shown. The leaf descriptions contain the sequence ID, genus and taxonomic placement. (PDF $4260 \mathrm{~kb}$ ) 
Additional file 8: Amino acid sequence alignment of E. nipponicum cathepsin B with cathepsins B of S. mansoni. Whole zymogens including a signal peptide were aligned. Numbers at the end of the lines show amino acid numbering of the mature parts of particular enzymes. Position of the pro-region cleavage site is marked by an arrow. The catalytic triad of the active site $(\mathrm{C}, \mathrm{H}, \mathrm{N})$ is marked by triangles. Conserved motifs around active site residues are shaded in grey. Tripeptides of potential $\mathrm{N}$-glycosylation sites are boxed. Predicted O-glycosylated residues are marked by grey squares. The occluding loop typical of cathepsins B is underlined. The 'haemoglobinase motif' ascribed to cathepsins B with an assumed function in haemoglobinolysis in blood-feeding helminths, is shaded in black (it is modified in SmCB2 and $E n(B)$. Residues within the $\mathrm{S} 2$ subsite of the active site involved in determining the substrate specificity are marked by a black dot and indicated with number (papain numbering). The alignment was made using sequences of Schistosoma mansoni cathepsins B1 (GenBank: CAD44624.1) and B2 (GenBank: XP_018651608.1). (PDF 1923 kb)

\section{Abbreviations}

AMC: 7-amino-4-methylcoumarin; BSA: Bovine serum albumin; Bz-Gly-HisLeu: Benzoyl-glycinyl-histidinyl-leucine; CA-074: N-[L-3-trans-propylcarbamoyloxirane2-carbonyl]-lle-Pro-OH; CBB: Coomassie Brilliant Blue; CPB: Citrate/phosphate buffer; DCG-04: fluorescent labelled probe, derivate of inhibitor E-64; DTT: Dithiothreitol; E-64: (L-trans-epoxysuccinyl-leucylamido [4-guanidino] butane); EnCB: Eudiplozoon nipponicum cathepsin B; EnCL: Eudiplozoon nipponicum cathepsin L; ESP: Excretory secretory products; FPLC: Fast protein liquid chromatography; FR: Substrate for serine and cysteine peptidases Z-Phe-Arg-AMC; GPR: Substrate for serine peptidases Z-Gly-Pro-Arg-AMC; His-tag: polyhistidine-tag (6xHis); ICL: Reversible inhibitor of cathepsin L(Arg-Lys-Leu-Leu-Trp-NH2); LR: Substrate for serine and cysteine peptidases Z-Leu-Arg-AMC; MES: (2-N-morpholino) ethanesulfonic acid; Ni-NTA: Nickel-nitrilotriacetic acid; PBS- TX100: Phosphate buffered saline containing 0.3\% Triton X100; PBS: Phosphate buffered saline;

PBS-T: Phosphate-buffered saline containing 0.05\% Tween 20; PR: Substrate for serine peptidases Z-Pro-Arg-AMC; PVDF: Polyvinylidene difluoride;

RACE-PCR: Rapid amplification of CDNA ends; RR: Substrate for cathepsin B Z-Arg-Arg-AMC; RT: Room temperature; RT-PCR: Reverse transcription-PCR; SDS-PAGE: Sodium dodecyl sulfate polyacrylamide gel electrophoresis; SSC: Saline-sodium citrate buffer

\section{Acknowledgements}

We would like to express our sincere gratitude to Professor John P. Dalton, Medical Biology Centre, School of Biological Sciences, Queen's University Belfast, for enabling $L$ to make use of his institute's experimental facilities, for valuable advice offered to $\sqcup$ during her scientific stay, and for critical comments on the final version of the manuscript. We wish to thank Professor Jan Konvalinka and Dr Tomáš Knedlík, Institute of Organic Chemistry and Biochemistry, ASCR, Praha, for providing us with iBody4. We would also like to thank Dr Conor R. Caffrey and Dr Doron C. Greenbaum, UCSD, Skaggs School of Pharmacy \& Pharmaceutical Sciences, CA, USA, who kindly provided the Green BODIPY-DCG-04 probe. Our gratitude also extends to Mr Stanislav Sojka, Rybářství Treboň, Plc., for arrangements concerning the collection of parasites. Computational resources were provided by the CESNET LM2015042 and the CERIT Scientific Cloud LM2015085, as part of the 'Projects of Large Research, Development, and Innovations Infrastructures' programme.

\section{Funding}

This work was supported by the project "Centre for Research of Pathogenicity and Virulence of Parasites" (No. CZ.02.1.01/0.0/0.0/16_019/0000759) funded by European Regional Development Fund and Ministry of Education, Youth and Sports of the Czech Republic. The research was also funded by Czech Science Foundation grants (P506/12/1258, GBP505/12/G112), Charles University institutional support (PROGRES Q43, UNCE 204017, and SW 244-260432/2017), Masaryk University (MUNI/A/1362/2016), Ministry of Education, Youth, and Sports of the Czech Republic (InterBioMed LO1302), and by the IOCB institutional project (RVO 61388963).

\section{Availability of data and materials}

Transcripts from the assembled transcriptome of adult E. nipponicum worms have been deposited at DDBJ/EMBL/GenBank under the accession GFYM00000000. The version described in this paper is the first version, GFYM01000000.

\section{Authors' contributions}

$\mathrm{L}$ produced recombinant cathepsins $\mathrm{L}$, performed their functional characterisation, in situ hybridisation and immunohistochemistry, participated in the bioinformatic annotation of enzyme structures, data interpretation, and in manuscript writing. HD produced the recombinant cathepsin B, performed its functional characterisation and immunohistochemistry, participated in the bioinformatic annotation of enzyme structure, data interpretation, and in manuscript writing. JD participated in the expression of recombinant cathepsins $L$ and their biochemical characterisation. MK took part in designing the study and in data interpretation. LU was involved in designing of RNA probes and in the process of in situ hybridization. JV assembled the transcriptome, mined the sequence data and evaluated expression rates of the enzymes. VZ performed the phylogenetic analyses. LM conducted the work, prepared the study design, and participated in the purification of recombinant enzymes, bioinformatic annotation, interpretation of data, and in the production of the final draft of the manuscript. All authors read and approved the final manuscript.

\section{Ethics approval and consent to participate}

The maintenance and care of experimental animals was carried out in accordance with European Directive 2010/63/EU, and according to Czech law (246/1992 and 359/2012) for biomedical research involving animals. Experiments were performed with a legal consent of the relevant institutional animal research ethics committees, namely the Professional Ethics Committee of the Faculty of Science, Charles University in Prague and the Branch for Research and Development of the Ministry of Education, Youth and Sports of the Czech Republic. The animal facility, its equipment, animal welfare and accompanying services, including maintenance of experimental animals, have been approved by the Branch of Animal Commodities of the Ministry of Agriculture of the Czech Republic (approval no. 13060/2014-MZE-17214)

\section{Consent for publication}

Not applicable.

\section{Competing interests}

The authors declare that they have no competing interests.

\section{Publisher's Note}

Springer Nature remains neutral with regard to jurisdictional claims in published maps and institutional affiliations.

\section{Author details}

1'Department of Parasitology, Faculty of Science, Charles University, Viničná 7, 12844 Prague 2, Czech Republic. ${ }^{2}$ Medical Biology Centre, School of Biological Sciences, Queen's University Belfast, 97 Lisburn Road, Belfast BT9 7BL, UK. ${ }^{3}$ Department of Zoology and Fisheries, Faculty of Agrobiology, Food and Natural Resources, Czech University of Life Sciences Praque, Kamýcká 129, 16500 Prague 6, Czech Republic. ${ }^{4}$ Department of Botany and Zoology, Faculty of Science, Masaryk University, Kotlárská 2, 61137 Brno, Czech Republic. Institute of Organic Chemistry and Biochemistry, Academy of Sciences of the Czech Republic, Flemingovo nám. 2, 16000 Prague 6, Czech Republic. ${ }^{6}$ Department of Parasitology, Faculty of Science, Charles University, Průmyslová 595, Vestec 25250, Czech Republic.

Received: 14 September 2017 Accepted: 22 January 2018 Published online: 06 March 2018

\section{References}

1. Denis A, Gabron C, Lambert A. Presence en France du deux parasites d'origine Est-asiatique: Diplozoon nipponicum Goto 1891 et Bothriocephalus acheilognathi Yamaguti, 1934 (Cestoda) chez Cyprinus carpio (Teleostei, Cyprinidae). Bull Fr Piscic. 1983;289:128-34.

2. Matějusová I, Koubková B, D’ Amelio S, Cunningham CO. Genetic characterization of six species of diplozoids (Monogenea; Diplozoidae). Parasitology. 2001;123:465-74.

3. Fisheries and Aquaculture Department. About us - Fisheries and Aquaculture Department.. http://www.fao.org/fishery/about/en Updated 17 March 2017.

4. Kawatsu H. Studies on the anemia of fish-IX, hypochromic microcytic anemia of crucian carp caused by infestation with a trematode, Diplozoon nipponicum. Bull Japan Soc Sci fish. 1978;44:1315-9. 
5. Smyth JD, Halton DW. The Monogenean - functional morphology. In: Smyth JD, Halton DW, editors. The Physiology of Trematodes. Cambridge: Cambridge University Press; 1983. p. 250-88.

6. Smyth JD, Halton DW. The Monogenean - physiology. In: Smyth JD, Halton DW, editors. The Physiology of Trematodes. Cambridge: Cambridge University Press; 1983. p. 289-320.

7. Hodová I, Matějusová I, Gelnar M. The surface topography of Eudiplozoon nipponicum (Monogenea) developmental stages parasitizing carp (Cyprinus carpio L.). Cent Eur J Biol. 2010;5:702-9.

8. Valigurová A, Hodová I, Sonnek R, Koubková B, Gelnar M. Eudiplozoon nipponicum in focus: monogenean exhibiting a highly specialized adaptation for ectoparasitic lifestyle. Parasitol Res. 2011;108:383-94.

9. Konstanzová V, Koubková B, Kašný M, llgová J, Dzika E, Gelnar M. Ultrastructure of the digestive tract of Paradiplozoon homoion (Monogenea) Parasitol Res. 2015;114:1485-94

10. Sonenshine D. Biology of ticks. Volume 1. New York: Oxford University Press; 1991.

11. Sojka D, Franta Z, Horn M, Caffrey CR, Mareš M, Kopáček P. New insights into the machinery of blood digestion by ticks. Trends Parasitol. 2013;29: 276-85.

12. Jedličková L, Dvořáková $H$, Kašný $M$, Ilgová J, Potěšil $D, Z$ dráhal Z, et al. Major acid endopeptidases of the blood-feeding monogenean Eudiplozoon nipponicum (Heteronchoinea: Diplozoidae). Parasitology. 2016;143:494-506.

13. Sajid M, JH MK. Cysteine proteases of parasitic organisms. Mol Biochem Parasitol. 2002;120:1-21.

14. Williamson AL, Brindley PJ, Knox DP, Hotez PJ, Loukas A. Digestive proteases of blood-feeding nematodes. Trends Parasitol. 2003;19:417-23.

15. Williamson AL, Lecchi P, Turk BE, Choe Y, Hotez PJ, JH MK, et al. A multi-enzyme cascade of hemoglobin proteolysis in the intestine of blood-feeding hookworms. J Biol Chem. 2004;279:35950-7.

16. Delcroix M, Sajid M, Caffrey CR, Lim KC, Dvořák J, Hsieh I, et al. A multienzyme network functions in intestinal protein digestion by a platyhelminth parasite. J Biol Chem. 2006;281:39316-29.

17. Atkinson H, Babbitt P, Sajid M. The global cysteine peptidase landscape in parasites. Trends Parasitol. 2009;25:573-81.

18. Kašný M, Mikeš L, Hampl V, Dvořák J, Caffrey CR, Dalton JP, et al. Peptidases of trematodes. Adv Parasitol. 2009:69:205-97.

19. Robinson MW, Dalton JP. Cysteine proteases of pathogenic organisms (Vol. 712). Boston: Springer; 2011.

20. McVeigh P, Maule AG, Dalton JP, Robinson MW. Fasciola hepatica virulenceassociated cysteine peptidases: a systems biology perspective. Microbes Infect. 2012;14:301-10.

21. NCBI Resource Coordinators NR. Database resources of the National Center for Biotechnology Information. Nucleic Acids Res. 2016;44:D7-19.

22. Rawlings ND, Barrett AJ, Finn R. Twenty years of the MEROPS database of proteolytic enzymes, their substrates and inhibitors. Nucleic Acids Res. 2016; 44:D343-50.

23. The UniProt Consortium. UniProt: the universal protein knowledgebase. Nucleic Acids Res. 2017;45:D158-69.

24. Boutet E, Lieberherr D, Tognolli M, Schneider M, Bansal P, Bridge AJ, et al. UniProtKB/Swiss-Prot, the manually annotated section of the UniProt KnowledgeBase: how to use the entry view. Methods Mol Biol. 2016;1374:23-54.

25. Berman HM, Westbrook J, Feng Z, Gilliland G, Bhat TN, Weissig H, et al. The protein data bank. Nucleic Acids Res. 2000;28:235-42.

26. Mashima J, Kodama Y, Kosuge T, Fujisawa T, Katayama T, Nagasaki H, et al. DNA data bank of Japan (DDBJ) progress report. Nucleic Acids Res. 2016;44: D51-7.

27. Sievers F, Wilm A, Dineen D, Gibson TJ, Karplus K, Li W, et al. Fast, scalable generation of high-quality protein multiple sequence alignments using Clustal omega. Mol Syst Biol. 2011;7:539.

28. Bray NL, Pimentel H, Melsted P, Pachter L. Near-optimal probabilistic RNA-seq quantification. Nat Biotechnol. 2016;34:525-7.

29. Petersen TN, Brunak S, von Heijne G, Nielsen H. SignalP 4.0: discriminating signal peptides from transmembrane regions. Nat Methods. 2011;8:785-6.

30. Artimo P, Jonnalagedda M, Arnold K, Baratin D, Csardi G, de Castro E, et al. EXPASy: SIB bioinformatics resource portal. Nucleic Acids Res. 2012;40:W597-603.

31. Steentoft C, Vakhrushev SY, Joshi HJ, Kong Y, Vester-Christensen MB, KT-BG $\mathrm{S}$, et al. Precision mapping of the human O-GalNAc glycoproteome through Simple Cell technology. EMBO J. 2013;32:1478-88.

32. Pruitt KD, Tatusova T, Maglott DR. NCBI reference sequences (RefSeq): a curated non-redundant sequence database of genomes, transcripts and proteins. Nucleic Acids Res. 2007;35:D61-5.
33. Howe KL, Bolt BJ, Shafie M, Kersey P, Berriman M. WormBase ParaSite - a comprehensive resource for helminth genomics. Mol Biochem Parasitol. 2017;215:2-10.

34. Frickey T, Lupas A. CLANS: a Java application for visualizing protein families based on pairwise similarity. Bioinformatics. 2004;20:3702-4.

35. Li W, Godzik A. Cd-hit: a fast program for clustering and comparing large sets of protein or nucleotide sequences. Bioinformatics. 2006;22:1658-9.

36. Katoh K, Standley DM. MAFFT multiple sequence alignment software version 7: improvements in performance and usability. Mol Biol Evol. 2013; 30:772-80.

37. Criscuolo A, Gribaldo S. BMGE (block mapping and gathering with entropy): a new software for selection of phylogenetic informative regions from multiple sequence alignments. BMC Evol Biol. 2010;10:210.

38. Nguyen L-T, Schmidt HA, von Haeseler A, Minh BQ. IQ-TREE: a fast and effective stochastic algorithm for estimating maximum-likelihood phylogenies. Mol Biol Evol. 2015;32:268-74.

39. Caffrey CR, Salter JP, Lucas KD, Khiem D, Hsieh I, Lim K-C, et al. SmCB2, a novel tegumental cathepsin B from adult Schistosoma mansoni. Mol Biochem Parasitol. 2002;121:49-61.

40. Dvořák J, Delcroix M, Rossi A, Vopálenský V, Pospišek M, Šedinová M, et al. Multiple cathepsin B isoforms in schistosomula of Trichobilharzia regenti: identification, characterisation and putative role in migration and nutrition. Int J Parasitol. 2005;35:895-910.

41. Wang B-L, Xu Y, Wu C-Q, Xu Y-M, Wang H-H. Cloning, expression, and refolding of a secretory protein ESAT-6 of Mycobacterium tuberculosis. Protein Expr Purif. 2005;39:184-8.

42. Greenbaum D, Baruch A, Hayrapetian L, Darula Z, Burlingame A, Medzihradszky KF, et al. Chemical approaches for functionally probing the proteome. Mol Cell Proteomics. 2002;1:60-8.

43. Sajid M, JH MK, Hansell E, Mathieu MA, Lucas KD, Hsieh I, et al. Functional expression and characterization of Schistosoma mansoni cathepsin B and its trans-activation by an endogenous asparaginyl endopeptidase. Mol Biochem Parasitol. 2003;131:65-75.

44. Quack T, Knobloch J, Beckmann S, Vicogne J, Dissous C, Grevelding CG. The formin-homology protein SmDia interacts with the Src Kinase SmTK and the GTPase SmRho1 in the gonads of Schistosoma mansoni. PLoS One. 2009:4: e6998.

45. ECH H, Donaldson ME, Saville BJ. Detection of antisense RNA transcripts by strand-specific RT-PCR. Methods Mol Biol. 2010;630:125-38.

46. Šácha P, Knedlík T, Schimer J, Tykvart J, Parolek J, Navrátil V, et al. iBodies: modular synthetic antibody mimetics based on hydrophilic polymers decorated with functional moieties. Angew Chem Int Ed Engl. 2016;55: 2356-60.

47. Baig S, Damian RT, Peterson DS. A novel cathepsin B active site motif is shared by helminth bloodfeeders. Exp Parasitol. 2002;101:83-9.

48. Tort J, Brindley PJ, Knox D, Wolfe KH, Dalton JP. Proteinases and associated genes of parasitic helminths. Adv Parasitol. 1999:43:161-266.

49. Dalton JP, Caffrey CR, Sajid M, Stack C, Donnelly S, Loukas A, et al. Proteases in trematode biology. In: Maule A, Marks N, editors. Parasitic flatworms: molecular biology, biochemistry, immunology and physiology. Cambridge: CABl; 2006. p. 348-68.

50. Smooker PM, Whisstock JC, Irving JA, Siyaguna S, Spithill TW, Pike RN. A single amino acid substitution affects substrate specificity in cysteine proteinases from Fasciola hepatica. Protein Sci. 2000;9:2567-72.

51. Stack CM, Caffrey CR, Donnelly SM, Seshaadri A, Lowther J, Tort JF, et al. Structural and functional relationships in the virulence-associated cathepsin L proteases of the parasitic liver fluke, Fasciola hepatica. J Biol Chem. 2008; 283:9896-908.

52. Norbury $\sqcup$, Hung A, Beckham S, Pike RN, Spithill TW, Craik CS, et al. Analysis of Fasciola cathepsin $L 5$ by $S 2$ subsite substitutions and determination of the P1-P4 specificity reveals an unusual preference. Biochimie. 2012;94:1119-27.

53. Pillay D, Boulangé AF, THT C. Expression, purification and characterisation of two variant cysteine peptidases from Trypanosoma congolense with active site substitutions. Protein Expr Purif. 2010;74:264-71.

54. Lecaille F, Choe Y, Brandt W, Li Z, Craik CS, Brömme D. Selective inhibition of the collagenolytic activity of human cathepsin $\mathrm{K}$ by altering its $\mathrm{S} 2$ subsite specificity. Biochemistry. 2002;41:8447-54.

55. Corvo I, Cancela M, Cappetta M, Pi-Denis N, Tort JF, Roche L. The major cathepsin L secreted by the invasive juvenile Fasciola hepatica prefers proline in the S2 subsite and can cleave collagen. Mol Biochem Parasitol. 2009;167:41-7. 
56. Choe Y, Leonetti F, Greenbaum DC, Lecaille F, Bogyo M, Brömme D, et al. Substrate profiling of cysteine proteases using a combinatorial peptide librany identifies functionally unique specificities. J Biol Chem. 2006;281:12824-32.

57. Brömmes D, Bonneaug PR, Lachance $P$, Storer AC. Engineering the S2 subsite specificity of human cathepsin S to a cathepsin L-and cathepsin Blike specificity. J Biol Chem. 1994;269:30238-42.

58. Lartillot N, Brinkmann H, Philippe H. Suppression of long-branch attraction artefacts in the animal phylogeny using a site-heterogeneous model. BMC Evol Biol. 2007;7:S4.

59. Dolečková K, Kašný M, Mikeš L, Cartwright J, Jedelský P, Schneider EL, et al. The functional expression and characterisation of a cysteine peptidase from the invasive stage of the neuropathogenic schistosome Trichobilharzia regenti. Int J Parasitol. 2009;39:201-11.

60. McGrath ME. The lysosomal cysteine proteases. Annu Rev Biophys Biomol Struct. 1999;28:181-204.

61. Mikeš L, Man P. Purification and characterization of a saccharide-binding protein from penetration glands of Diplostomum pseudospathaceum - a bifunctional molecule with cysteine protease activity. Parasitology. 2003;127: 69-77.

62. Brady CP, Brindley PJ, Dowd AJ, Dalton JP. Schistosoma mansoni: differential expression of cathepsins L1 and L2 suggests discrete biological functions for each enzyme. Exp Parasitol. 2000;94:75-83.

63. Dvořák J, Mashiyama ST, Sajid M, Braschi S, Delcroix M, Schneider EL, et al. SmCL3, a gastrodermal cysteine protease of the human blood fluke Schistosoma mansoni. PLoS Negl Trop Dis. 2009;3:e449.

64. Lowther J, Robinson MW, Donnelly SM, Xu W, Stack CM, Matthews JM, et al. The importance of $\mathrm{pH}$ in regulating the function of the Fasciola hepatica cathepsin L1 cysteine protease. PLoS Negl Trop Dis. 2009;3:e369.

65. Franta Z, Sojka D, Frantová H, Dvořák J, Horn M, Srba J, et al. IrCL1 - the haemoglobinolytic cathepsin L of the hard tick, Ixodes ricinus. Int J Parasitol. 2011:41:1253-62.

66. Brinker A, Weber E, Stoll D, Voigt J, Müller A, Sewald N, et al. Highly potent inhibitors of human cathepsin $\mathrm{L}$ identified by screening combinatorial pentapeptide amide collections. Eur J Biochem. 2000;267:5085-92

67. Steverding D. The cathepsin B-selective inhibitors CA-074 and CA-074Me inactivate cathepsin $L$ under reducing conditions. Open Enzym Inhib J. 2011;4:11-6.

68. Steverding D, Sexton DW, Wang X, Gehrke SS, Wagner GK, Caffrey CR. Trypanosoma brucei: chemical evidence that cathepsin $L$ is essential for survival and a relevant drug target. Int J Parasitol. 2012;42:481-8.

69. Mindell JA. Lysosomal acidification mechanisms. Annu Rev Physiol. 2012;74: 69-86.

70. Chappell CL, Dresden MH. Schistosoma mansoni: Proteinase activity of "hemoglobinase" from the digestive tract of adult worms. Exp Parasitol. 1986;61:160-7

71. Halton DW. Nutritional adaptations to parasitism within the Platyhelminthes. Int J Parasitol. 1997;27:693-704.

72. Dalton JP, Skelly P, Halton DW. Role of the tegument and gut in nutrient uptake by parasitic platyhelminths. Can J Zool. 2004;82:211-32.

73. Robinson MW, Corvo I, Jones PM, George AM, Padula MP, To J, et al. Collagenolytic activities of the major secreted cathepsin $L$ peptidases involved in the virulence of the helminth pathogen, Fasciola hepatica. PLoS Negl Trop Dis. 2011;5:e1012.

74. Ozsolak F, Kapranov P, Foissac S, Kim SW, Fishilevich E, Monaghan AP, et al. Comprehensive polyadenylation site maps in yeast and human reveal pervasive alternative polyadenylation. Cell. 2010;143:1018-29.

75. Osato N, Yamada H, Satoh K, Ooka H, Yamamoto M, Suzuki K, et al. Antisense transcripts with rice full-length cDNAs. Genome Biol. 2003;5:R5.

76. Steigele $\mathrm{S}$, Nieselt K. Open reading frames provide a rich pool of potential natural antisense transcripts in fungal genomes. Nucleic Acids Res. 2005;33: 5034-44.

77. Henke C, Strissel PL, Schubert M-T, Mitchell M, Stolt CC, Faschingbauer F, et al. Selective expression of sense and antisense transcripts of the sushi-ichirelated retrotransposon-derived family during mouse placentogenesis. Retrovirology. 2015;12:9.

78. Pelechano V, Steinmetz LM. Gene regulation by antisense transcription. Nat Rev Genet. 2013;14:880-93.

79. Illy C, Quraishi O, Wang J, Purisima E, Vernet T, Mort JS. Role of the occluding loop in cathepsin B activity. J Biol Chem. 1997;272:1197-202.
80. Michel A, Ghoneim H, Resto M, Klinkert MQ, Kunz W. Sequence, characterization and localization of a cysteine proteinase cathepsin $L$ in Schistosoma mansoni. Mol Biochem Parasitol. 1995;73:7-18.

81. Britton C, Murray L. A cathepsin L protease essential for Caenorhabditis elegans embryogenesis is functionally conserved in parasitic nematodes. Mol Biochem Parasitol. 2002;122:21-33.

82. Pinlaor P, Kaewpitoon N, Laha T, Sripa B, Kaewkes S, Morales ME, et al. Cathepsin F cysteine protease of the human liver fluke. PLoS Negl Trop Dis. 2009;3:e398.

83. Ogawa K. Diseases of cultured marine fishes caused by Platyhelminthes (Monogenea, Digenea, Cestoda). Parasitology. 2015;142:178-95.

\section{Submit your next manuscript to BioMed Central and we will help you at every step:}

- We accept pre-submission inquiries

- Our selector tool helps you to find the most relevant journal

- We provide round the clock customer support

- Convenient online submission

- Thorough peer review

- Inclusion in PubMed and all major indexing services

- Maximum visibility for your research

Submit your manuscript at www.biomedcentral.com/submit
Biomed Central 